\title{
Volume, Polar Volume and Euler Characteristic for Convex Functions
}

\author{
Fabian Mussnig
}

\begin{abstract}
Functional analogs of the Euler characteristic and volume together with a new analog of the polar volume are characterized as non-negative, continuous, $\operatorname{SL}(n)$ and translation invariant valuations on the space of finite, convex and coercive functions on $\mathbb{R}^{n}$.
\end{abstract}

2010 AMS subject classification: 26B25 (46A40, 52A20, 52A41, 52B45).

\section{Introduction and Statement of the Main Result}

A map $\mathrm{Z}$ defined on the subset $\mathcal{S}$ of a lattice $(\mathcal{L}, \vee, \wedge)$ and taking values in an abelian semigroup is called a valuation if

$$
\mathrm{Z}(u \vee v)+\mathrm{Z}(u \wedge v)=\mathrm{Z}(u)+\mathrm{Z}(v)
$$

whenever $u, v, u \vee v, u \wedge v \in \mathcal{S}$. Valuations defined on the set of convex bodies (compact convex sets), $\mathcal{K}^{n}$, in $\mathbb{R}^{n}$ have been studied since Dehn's solution of Hilbert's Third Problem in 1901. In this case, $\vee$ and $\wedge$ denote union and intersection, respectively, and the set $\mathcal{K}^{n}$ is equipped with the topology induced by the Hausdorff metric. The first classification of valuations on convex bodies and a characterization of the Euler characteristic, $V_{0}$, and the $n$-dimensional volume (that is, the Lebesgue measure), $V_{n}$, was obtained by Blaschke [8].

Theorem 1.1 (Blaschke). For $n \geq 2$, a map $\mu: \mathcal{K}^{n} \rightarrow \mathbb{R}$ is a continuous, $\mathrm{SL}(n)$ and translation invariant valuation if and only if there exist constants $c_{0}, c_{1} \in \mathbb{R}$ such that

$$
\mu(K)=c_{0} V_{0}(K)+c_{1} V_{n}(K)
$$

for every $K \in \mathcal{K}^{n}$.

Here, a valuation $\mu: \mathcal{K}^{n} \rightarrow \mathbb{R}$ is called $\mathrm{SL}(n)$ invariant if $\mu(\phi K)=\mu(K)$ for all $\phi \in \mathrm{SL}(n)$ and $K \in \mathcal{K}^{n}$, where $\phi K=\{\phi x: x \in K\}$. Moreover, $\mu$ is said to be translation invariant if $\mu(K+x)=$ $\mu(K)$ for all $x \in \mathbb{R}^{n}$, with $K+x=\{y+x: y \in K\}$. See also [1 33, 7, 19, 21, 23, 27, 28, 34, 44] for some recent results on valuations on convex bodies and [22, 24] for more information on the classical theory.

More recently, valuations on function spaces have been introduced and studied. Here, $u \vee v$ denotes the pointwise maximum of $u$ and $v$ and $u \wedge v$ the pointwise minimum of two functions $u, v \in \mathcal{S}$, where $\mathcal{S}$ is a space of real-valued functions. The first results for valuations on Sobolev 
spaces were obtained by Ludwig [30, 32] and Ma [36]. For $L^{p}$ spaces complete classifications for valuations intertwining the $\mathrm{SL}(n)$ were established in [33, 46, 47]. Bobkov, Colesanti and Fragalà [9] as well as Milman together with Rotem [39] extended intrinsic volumes to the space of quasi-concave functions (see also [10,25]). A classification of rigid motion invariant valuations on quasi-concave functions was established by Colesanti and Lombardi [1] and for definable functions such a result was previously established by Baryshnikov, Ghrist and Wright [5]. For further results, see also [4, 12, 13, 26, 31, 40, 45, 48, 49].

For convex functions, a characterization of functional analogs of the Euler characteristic and volume was established in [15]. Let $\operatorname{Conv}\left(\mathbb{R}^{n}\right)$ denote the space of all convex functions $u: \mathbb{R}^{n} \rightarrow(-\infty,+\infty]$ that are proper, lower semicontinuous and coercive. Here a function $u$ is proper if it is not identically $+\infty$ and it is called coercive if

$$
\lim _{|x| \rightarrow+\infty} u(x)=+\infty
$$

Furthermore, the space $\operatorname{Conv}\left(\mathbb{R}^{n}\right)$ is equipped with the topology associated to epi-convergence (see also Section 3 ).

We say that a map Z, defined on a space of real-valued functions $\mathcal{S}$ on $\mathbb{R}^{n}$, is translation invariant if $\mathrm{Z}\left(u \circ \tau^{-1}\right)=\mathrm{Z}(u)$ for every $u \in \mathcal{S}$ and translation $\tau: \mathbb{R}^{n} \rightarrow \mathbb{R}^{n}$. Moreover, $\mathrm{Z}$ is called $\operatorname{SL}(n)$ invariant if $\mathrm{Z}\left(u \circ \phi^{-1}\right)=\mathrm{Z}(u)$ for every $u \in \mathcal{S}$ and $\phi \in \operatorname{SL}(n)$.

Theorem 1.2 ([15]). For $n \geq 2$, a map $\mathrm{Z}: \operatorname{Conv}\left(\mathbb{R}^{n}\right) \rightarrow[0, \infty)$ is a continuous, $\mathrm{SL}(n)$ and translation invariant valuation if and only if there exist continuous functions $\zeta_{0}, \zeta_{1}: \mathbb{R} \rightarrow[0, \infty)$ where $\zeta_{1}$ has finite moment of order $n-1$ such that

$$
\mathrm{Z}(u)=\zeta_{0}\left(\min _{x \in \mathbb{R}^{n}} u(x)\right)+\int_{\operatorname{dom} u} \zeta_{1}(u(x)) \mathrm{d} x
$$

for every $u \in \operatorname{Conv}\left(\mathbb{R}^{n}\right)$.

Here, a function $\zeta: \mathbb{R} \rightarrow[0, \infty)$ has finite moment of order $n-1$ if $\int_{0}^{+\infty} t^{n-1} \zeta(t) \mathrm{d} t<+\infty$ and $\operatorname{dom} u$ denotes the domain of $u$, that is, $\operatorname{dom} u=\left\{x \in \mathbb{R}^{n}: u(x)<+\infty\right\}$. Furthermore, for functions $u \in \operatorname{Conv}\left(\mathbb{R}^{n}\right)$, the minimum is attained and hence finite.

The proof of this theorem uses the following classification of continuous and $\operatorname{SL}(n)$ invariant valuations on $\mathcal{K}_{o}^{n}$, the space of convex bodies which contain the origin. A functional $\mu: \mathcal{K}_{o}^{n} \rightarrow \mathbb{R}$ is a continuous and $\operatorname{SL}(n)$ invariant valuation if and only if there exist constants $c_{0}, c_{1} \in \mathbb{R}$ such that

$$
\mu(K)=c_{0} V_{0}(K)+c_{n} V_{n}(K)
$$

for every $K \in \mathcal{K}_{o}^{n}$ (see, for example, [35, Corollary 1.2]). However, if one restricts to the class $\mathcal{K}_{(o)}^{n}$ of convex bodies that contain the origin in their interiors, an additional $\mathrm{SL}(n)$ invariant valuation appears. Therefore, let

$$
K^{*}=\left\{x \in \mathbb{R}^{n}: x \cdot y \leq 1, \forall y \in K\right\}
$$

denote the polar set of $K \in \mathcal{K}^{n}$. If $K$ contains the origin in its interior, then also $K^{*}$ is an element of $\mathcal{K}_{(o)}^{n}$ and hence bounded. In this case, $K^{*}$ is also said to be the polar body of $K$. We 
now define $V_{n}^{*}(K)=V_{n}\left(K^{*}\right)$ as the polar volume of $K \in \mathcal{K}_{(o)}^{n}$, which is an important quantity in convex geometric analysis. For example the famous but still unsolved Mahler conjecture states that the minimum of $V_{n}^{*}(K)$ among all origin-symmetric bodies $K \in \mathcal{K}_{(o)}^{n}$ with $V_{n}(K)=1$ is attained by a hypercube. The first characterization of the polar volume was obtained by Ludwig in [29]. More recently, a long conjectured classification similar to Theorem 1.1] was obtained by Haberl and Parapatits.

Theorem $1.3([20])$. For $n \geq 2$, a map $\mu: \mathcal{K}_{(o)}^{n} \rightarrow \mathbb{R}$ is a continuous and $\mathrm{SL}(n)$ invariant valuation if and only if there exist constants $c_{0}, c_{1}, c_{2} \in \mathbb{R}$ such that

$$
\mu(K)=c_{0} V_{0}(K)+c_{1} V_{n}(K)+c_{2} V_{n}^{*}(K),
$$

for every $K \in \mathcal{K}_{(o)}^{n}$.

In order to establish an analog of this result for convex functions, let

$$
u^{*}(x)=\sup _{y \in \mathbb{R}^{n}}(x \cdot y-u(y)), \quad x \in \mathbb{R}^{n}
$$

denote the convex conjugate of a function $u: \mathbb{R}^{n} \rightarrow[-\infty, \infty]$, where $x \cdot y$ denotes the inner product of $x, y \in \mathbb{R}^{n}$. If $u$ is proper and does not attain $-\infty$, then the function $u^{*}: \mathbb{R}^{n} \rightarrow$ $(-\infty,+\infty]$ is always convex, proper and lower semicontinuous. Furthermore, convex conjugation is a continuous operation and is compatible with $\mathrm{SL}(n)$ transforms (for details see Section 3 ). Let $\operatorname{Conv}\left(\mathbb{R}^{n}, \mathbb{R}\right)$ denote the space of all convex, coercive functions $u: \mathbb{R}^{n} \rightarrow \mathbb{R}$. We will prove the following result.

Theorem. For $n \geq 2$, a map $\mathrm{Z}: \operatorname{Conv}\left(\mathbb{R}^{n}, \mathbb{R}\right) \rightarrow[0, \infty)$ is a continuous, $\operatorname{SL}(n)$ and translation invariant valuation if and only if there exist continuous functions $\zeta_{0}, \zeta_{1}, \zeta_{2}: \mathbb{R} \rightarrow[0, \infty)$ where $\zeta_{1}$ has finite moment of order $n-1$ and $\zeta_{2}(t)=0$ for all $t \geq T$ with some $T \in \mathbb{R}$ such that

$$
\mathrm{Z}(u)=\zeta_{0}\left(\min _{x \in \mathbb{R}^{n}} u(x)\right)+\int_{\mathbb{R}^{n}} \zeta_{1}(u(x)) \mathrm{d} x+\int_{\operatorname{dom} u^{*}} \zeta_{2}\left(\nabla u^{*}(x) \cdot x-u^{*}(x)\right) \mathrm{d} x
$$

for every $u \in \operatorname{Conv}\left(\mathbb{R}^{n}, \mathbb{R}\right)$.

Here, $\nabla u$ denotes the gradient of a function $u$ defined on $\mathbb{R}^{n}$. Note, that by Rademacher's theorem (see for example [17, Theorem 3.1.6]) a convex function is differentiable almost everywhere on the interior of its domain.

Remark 1.4. If (1) is evaluated for a so-called cone function $\ell_{K} \in \operatorname{Conv}\left(\mathbb{R}^{n}, \mathbb{R}\right)$ with $K \in \mathcal{K}_{(o)}^{n}$, that is a function which is defined via its sublevel sets

$$
\left\{\ell_{K} \leq t\right\}:=\left\{x \in \mathbb{R}^{n}: \ell_{K}(x) \leq t\right\}=t K,
$$

for $t \geq 0$ and $\left\{\ell_{K} \leq t\right\}=\emptyset$ for $t<0$, then a linear combination of $V_{0}(K), V_{n}(K)$ and $V_{n}^{*}(K)$ is obtained.

Remark 1.5. For a function $u \in \operatorname{Conv}\left(\mathbb{R}^{n}, \mathbb{R}\right) \cap C^{2}\left(\mathbb{R}^{n}\right)$, the new term in (1) can be rewritten as

$$
\int_{\mathbb{R}^{n}} \zeta_{2}(u(x)) \operatorname{det}\left(\mathrm{D}^{2} u(x)\right) \mathrm{d} x
$$

where $\mathrm{D}^{2} u(x)$ is the Hessian matrix of $u$ and $\operatorname{det}\left(\mathrm{D}^{2} u(x)\right)$ denotes its determinant. This is also a special case of the so-called Hessian valuations that were introduced in [14].

In addition to the main result, we will study functional analogs of further $\operatorname{SL}(n)$ invariant valuations in Section 4.3 . 


\section{Valuations on Convex Bodies}

In this section we consider some basic results about valuations on convex bodies and constructions on polytopes. Let $\mathcal{P}^{n} \subset \mathcal{K}^{n}$ denote the set of convex polytopes and let $\mathcal{P}_{o}^{n}$ and $\mathcal{P}_{(o)}^{n}$ denote the subsets of polytopes that contain the origin and polytopes that contain the origin in their interiors, respectively. For general references, we refer to the books of Gruber [18] and Schneider [43.

A real-valued valuation $\mu$ defined on some subset $\mathcal{Q}^{n} \subseteq \mathcal{K}^{n}$ is said to be homogeneous of degree $i \in \mathbb{R}$ if $\mu(\lambda K)=\lambda^{i} \mu(K)$ for every $\lambda>0$ and $K \in \mathcal{Q}^{n}$. Furthermore, for $i \in \mathbb{N}, \mu$ is said to be $i$-simple if $\mu(K)=0$ for every $K \in \mathcal{Q}^{n}$ with $\operatorname{dim} K<i$. For example, the Euler characteristic $V_{0}$ is homogeneous of degree 0 and the $n$-dimensional volume $V_{n}$ is homogeneous of degree $n$ and $n$-simple. Moreover, we have for any $K \in \mathcal{K}_{(o)}^{n}$ and $\lambda>0$

$$
V_{n}^{*}(\lambda K)=V_{n}\left((\lambda K)^{*}\right)=V_{n}\left(\lambda^{-1} K^{*}\right)=\lambda^{-n} V_{n}^{*}(K),
$$

which shows that the polar volume is homogeneous of degree $-n$.

The next result is due to [37,38] (see also [43, Corollary 6.3.2., Theorem 6.3.5]).

Theorem 2.1 (McMullen decomposition). Let $\mu: \mathcal{K}^{n} \rightarrow \mathbb{R}$ be a translation invariant, continuous valuation. There exist continuous, translation invariant valuations $\mu_{0}, \ldots, \mu_{n}$ on $\mathcal{K}^{n}$ such that $\mu_{i}$ is homogeneous of degree $i$ and $i$-simple for $0 \leq i \leq n$ and

$$
\mu(\lambda K)=\sum_{i=0}^{n} \lambda^{i} \mu_{i}(K)
$$

for every $K \in \mathcal{K}^{n}$ and $\lambda \geq 0$. In particular, $\mu=\mu_{0}+\cdots+\mu_{n}$.

For the proof of our main result we will need some constructions on polytopes that will be used as level sets of certain convex functions. In the following, let $\left\{e_{1}, \ldots, e_{n}\right\}$ denote the standard basis of $\mathbb{R}^{n}$ and let $T^{n}$ denote the standard simplex in $\mathbb{R}^{n}$, that is

$$
T^{n}=\operatorname{conv}\left\{0, e_{1}, \ldots, e_{n}\right\},
$$

where conv denotes the convex hull. Furthermore, set $\bar{e}:=(1, \ldots, 1)^{t}$. For $\delta>0$ we set $T_{\delta}:=(1+2 \delta) T^{n}-\delta \bar{e} \in \mathcal{P}^{n}$ or equivalently

$$
T_{\delta}=\operatorname{conv}\left\{\left(\begin{array}{c}
-\delta \\
-\delta \\
\vdots \\
-\delta
\end{array}\right),\left(\begin{array}{c}
1+\delta \\
-\delta \\
\vdots \\
-\delta
\end{array}\right), \cdots,\left(\begin{array}{c}
-\delta \\
\vdots \\
-\delta \\
1+\delta
\end{array}\right)\right\} .
$$

Note, that if $n=2$ or $n \geq 3$ and $0<\delta<\frac{1}{n-2}$, then $T_{\delta}$ contains the origin in its interior.

Lemma 2.2. Let $0<\delta<\frac{1}{n-2}$ if $n \geq 3$ and $0<\delta<1$ if $n=2$. For $b>0,0<\rho<1$ and $t \geq b$ let $x_{\delta}=(1+\delta,-\delta, \cdots,-\delta)^{t}$ and let $P_{\delta, \rho}^{b, t}:=t T_{\delta} \cap\left\{x_{1} \leq b(1+\delta)+\rho(t-b)\right\}$. It holds that

$$
\begin{aligned}
& P_{\delta, \rho}^{b, t} \cup\left((t-b) T_{\delta}+b x_{\delta}\right)=t T_{\delta} \\
& P_{\delta, \rho}^{b, t} \cap\left((t-b) T_{\delta}+b x_{\delta}\right)=(t-b)\left(T_{\delta} \cap\left\{x_{1} \leq \rho\right\}\right)+b x_{\delta}
\end{aligned}
$$


for every $t \geq b$. Furthermore

$$
V_{n}^{*}\left(T_{\delta} \cap\left\{x_{1} \leq \rho\right\}\right)=V_{n}^{*}\left(T_{\delta}\right)+\frac{1}{n ! \delta^{n-2}} \frac{1+\delta}{\delta(1-(n-2) \delta)}\left(\frac{1}{\rho}-\frac{1}{1+\delta}\right) .
$$

Proof. We will show identities (2) and (3) by adding the vector $\delta t \bar{e}$ first. Note, that by definition

$$
s T_{\delta}+\delta s \bar{e}=s c_{\delta} T^{n},
$$

for any $s>0$, where $c_{\delta}=(1+2 \delta)$. Therefore

$$
P_{\delta, \rho}^{b, t}+\delta t \bar{e}=t c_{\delta} T^{n} \cap\left\{x_{1} \leq b(1+\delta)+\delta t+\rho(t-b)\right\} .
$$

Furthermore,

$$
\begin{aligned}
b x_{\delta}+\delta t \bar{e} & =(b(1+\delta)+\delta t, \delta(t-b), \ldots, \delta(t-b))^{t} \\
& =\delta(t-b) \bar{e}+b c_{\delta} e_{1}
\end{aligned}
$$

which shows that

$$
\begin{aligned}
(t-b) T_{\delta}+b x_{\delta}+\delta t \bar{e} & =(t-b) T_{\delta}+\delta(t-b) \bar{e}+b c_{\delta} e_{1} \\
& =(t-b) c_{\delta} T^{n}+b c_{\delta} e_{1} .
\end{aligned}
$$

Hence, using (41) again, equation (2) is equivalent to

$$
\left(t c_{\delta} T^{n} \cap\left\{x_{1} \leq b(1+\delta)+\delta t+\rho(t-b)\right\}\right) \cup\left((t-b) c_{\delta} T^{n}+b c_{\delta} e_{1}\right)=t c_{\delta} T^{n},
$$

which follows from the intercept theorem and the fact that

$$
b(1+\delta)+\delta t+\rho(t-b) \geq b(1+2 \delta)=b c_{\delta} .
$$

Moreover,

$$
\begin{aligned}
(t-b)\left(T_{\delta} \cap\left\{x_{1} \leq \rho\right\}\right)+b x_{\delta} & +\delta t \bar{e} \\
& =\left((t-b) T_{\delta} \cap\left\{x_{1} \leq \rho(t-b)\right\}\right)+\delta(t-b) \bar{e}+b c_{\delta} e_{1} \\
& =\left((t-b) c_{\delta} T^{n}+b c_{\delta} e_{1}\right) \cap\left\{x_{1} \leq b(1+\delta)+\delta t+\rho(t-b)\right\} .
\end{aligned}
$$

This shows that (3) is equivalent to

$$
\begin{array}{r}
\left(t c_{\delta} T^{n} \cap\left\{x_{1} \leq b(1+\delta)+\delta t+\rho(t-b)\right\}\right) \cap\left((t-b) c_{\delta} T^{n}+b c_{\delta} e_{1}\right) \\
=\left((t-b) c_{\delta} T^{n}+b c_{\delta} e_{1}\right) \cap\left\{x_{1} \leq b(1+\delta)+\delta t+\rho(t-b)\right\}
\end{array}
$$

which is easy to see.

In order to show the second statement, note that

$$
T_{\delta}=\left\{x \cdot(1,0, \ldots, 0)^{t} \leq-\delta\right\} \cap \ldots \cap\left\{x \cdot(0, \ldots, 0,1)^{t} \leq-\delta\right\} \cap\left\{x \cdot(1, \ldots, 1)^{t} \leq 1-(n-2) \delta\right\} .
$$


Hence,

$$
T_{\delta}^{*}=\operatorname{conv}\left\{\left(\begin{array}{c}
-1 / \delta \\
0 \\
\vdots \\
0
\end{array}\right),\left(\begin{array}{c}
0 \\
-1 / \delta \\
\vdots \\
0
\end{array}\right), \cdots,\left(\begin{array}{c}
0 \\
0 \\
\vdots \\
-1 / \delta
\end{array}\right),\left(\begin{array}{c}
1 /(1-(n-2) \delta) \\
1 /(1-(n-2) \delta) \\
\vdots \\
1 /(1-(n-2) \delta)
\end{array}\right)\right\}
$$

Furthermore it is easy to see that $\left(T_{\delta} \cap\left\{x_{1} \leq \rho\right\}\right)^{*}=\operatorname{conv}\left\{T_{\delta}^{*},(1 / \rho, 0, \ldots, 0)^{t}\right\}$. Hence,

$$
V_{n}^{*}\left(T_{\delta} \cap\left\{x_{1} \leq \rho\right\}\right)=V_{n}^{*}\left(T_{\delta}\right)+V_{n}\left(K_{\delta, \rho}\right)
$$

with

$$
K_{\delta, \rho}=\operatorname{conv}\left\{\left(\begin{array}{c}
1 / \rho \\
0 \\
\vdots \\
0
\end{array}\right),\left(\begin{array}{c}
0 \\
-1 / \delta \\
\vdots \\
0
\end{array}\right), \cdots,\left(\begin{array}{c}
0 \\
0 \\
\vdots \\
-1 / \delta
\end{array}\right),\left(\begin{array}{c}
1 /(1-(n-2) \delta) \\
1 /(1-(n-2) \delta) \\
\vdots \\
1 /(1-(n-2) \delta)
\end{array}\right)\right\} .
$$

We use Laplace's formula (along the last column) to calculate $V_{n}\left(K_{\delta, \rho}\right)=V_{n}\left(K_{\delta, \rho}+(0, \ldots, 0,1 / \delta)^{t}\right)$ which is given by

$$
V_{n}\left(K_{\delta, \rho}\right)=\frac{1}{n !}\left|\operatorname{det}\left(\begin{array}{ccccc}
1 / \rho & 0 & \cdots & 0 & 1 /(1-(n-2) \delta) \\
0 & -1 / \delta & \cdots & 0 & 1 /(1-(n-2) \delta) \\
\vdots & \vdots & \ddots & \vdots & \vdots \\
0 & 0 & 0 & -1 / \delta & 1 /(1-(n-2) \delta) \\
1 / \delta & 1 / \delta & 1 / \delta & 1 / \delta & 1 /(1-(n-2) \delta)+1 / \delta
\end{array}\right)\right|
$$

This gives

$$
\begin{aligned}
n ! V_{n}\left(K_{\delta, \rho}\right)= & \mid(-1)^{n-1} \frac{1}{1-(n-2) \delta}(-1)^{n-1}\left(\frac{-1}{\delta}\right)^{n-1}+(-1)^{n} \frac{1}{1-(n-2) \delta} \frac{1}{\rho}(-1)^{n}\left(\frac{-1}{\delta}\right)^{n-2} \\
& +\cdots+\left(\frac{1}{1-(n-2) \delta}+\frac{1}{\delta}\right) \frac{1}{\rho}\left(\frac{-1}{\delta}\right)^{n-2} \mid \\
= & \left|\frac{1}{1-(n-2) \delta}\left(\frac{-1}{\delta}\right)^{n-1}+\frac{1}{\rho}\left(\frac{-1}{\delta}\right)^{n-2}\left(\frac{n-1}{1-(n-2) \delta}+\frac{1}{\delta}\right)\right| \\
= & \left|\left(\frac{-1}{\delta}\right)^{n-2} \frac{1+\delta}{\delta(1-(n-2) \delta)}\left(\frac{1}{\rho}-\frac{1}{1+\delta}\right)\right| \\
= & \frac{1}{\delta^{n-2}} \frac{1+\delta}{\delta(1-(n-2) \delta)}\left(\frac{1}{\rho}-\frac{1}{1+\delta}\right)
\end{aligned}
$$

which completes the proof.

In the following, we write $B^{n}=\left\{x \in \mathbb{R}^{n}:|x| \leq 1\right\}$ for the unit ball in $\mathbb{R}^{n}, Q^{n}=[-1,1]^{n}$ for the centered standard cube in $\mathbb{R}^{n}$ and

$$
C^{n}:=\operatorname{conv}\left\{ \pm e_{1}, \ldots, \pm e_{n}\right\}=\left(Q^{n}\right)^{*} \in \mathcal{P}_{(o)}^{n}
$$

for the corresponding cross-polytope. We will need the following result.

Lemma 2.3. Let $c_{i} \geq 0$ for $1 \leq i \leq n$ and let $K:=\operatorname{conv}\left\{0, c_{1} e_{1}, \ldots, c_{n} e_{n}\right\}$. For $\delta>0$, we have

$$
V_{n}\left(\operatorname{conv}\left(\delta C^{n} \cup K\right)\right)=\frac{1}{n !} \prod_{i=1}^{n}\left(\max \left\{c_{i}, \delta\right\}+\delta\right) .
$$


Proof. This follows easily if one considers that for $a_{i}, b_{i} \geq 0,1 \leq i \leq n$ one has

$$
V_{n}\left(\operatorname{conv}\left\{a_{1} e_{1},-b_{1} e_{1}, \ldots, a_{n} e_{n},-b_{n} e_{n}\right\}\right)=\frac{1}{n !} \prod_{i=1}^{n}\left(a_{i}+b_{i}\right),
$$

as well as

$$
\operatorname{conv}\left(\delta C^{n} \cup K\right)=\operatorname{conv}\left\{\max \left\{c_{1}, \delta\right\} e_{1},-\delta e_{1}, \ldots, \max \left\{c_{n}, \delta\right\} e_{n},-\delta e_{n}\right\}
$$

\section{Convex Functions}

We collect some results on convex functions. For basic references we refer to the books of Rockafellar \& Wets [42] and Schneider [43]. To every convex function $u: \mathbb{R}^{n} \rightarrow(-\infty,+\infty$ ] one can assign several convex sets. The (effective) domain of $u$, dom $u=\left\{x \in \mathbb{R}^{n}: u(x)<+\infty\right\}$, is a convex subset of $\mathbb{R}^{n}$ and the epigraph of $u$, epi $u=\left\{(x, y) \in \mathbb{R}^{n} \times \mathbb{R}: u(x) \leq y\right\}$, is a convex subset of $\mathbb{R}^{n} \times \mathbb{R}$. For $t \in \mathbb{R}$, the sublevel set,

$$
\{u \leq t\}=\left\{x \in \mathbb{R}^{n}: u(x) \leq t\right\},
$$

is convex. If $u$ is coercive, then its sublevel sets are bounded and if $u$ is lower semicontinuous the sublevel sets are closed. Hence, if $u \in \operatorname{Conv}\left(\mathbb{R}^{n}\right)$, the sets $\{u \leq t\}$ are elements of $\mathcal{K}^{n}$ for every $t \geq \min _{x \in \mathbb{R}^{n}} u(x)$. In particular, this minimum is attained and finite and the space $\operatorname{Conv}\left(\mathbb{R}^{n}\right)$ can be seen as a functional analog of $\mathcal{K}^{n}$. Note, that for $u, v \in \operatorname{Conv}\left(\mathbb{R}^{n}\right)$ and $t \in \mathbb{R}$

$$
\{u \wedge v \leq t\}=\{u \leq t\} \cup\{v \leq t\} \quad \text { and } \quad\{u \vee v \leq t\}=\{u \leq t\} \cap\{v \leq t\},
$$

where for $u \wedge v \in \operatorname{Conv}\left(\mathbb{R}^{n}\right)$ all occurring sublevel sets are either empty or in $\mathcal{K}^{n}$.

The space $\operatorname{Conv}\left(\mathbb{R}^{n}\right)$ is equipped with the topology associated to epi-convergence. A sequence $u_{k}: \mathbb{R}^{n} \rightarrow(-\infty,+\infty]$ is said to be epi-convergent to $u: \mathbb{R}^{n} \rightarrow(-\infty,+\infty]$ if for all $x \in \mathbb{R}^{n}$ the following conditions hold:

(i) For every sequence $x_{k}$ that converges to $x, u(x) \leq \liminf _{k \rightarrow+\infty} u_{k}\left(x_{k}\right)$.

(ii) There exists a sequence $x_{k}$ that converges to $x$ such that $u(x)=\lim _{k \rightarrow+\infty} u_{k}\left(x_{k}\right)$.

In other words, $u$ is an optimal common asymptotic lower bound of the sequence $u_{k}$. For epiconvergent sequences $u_{k}$ with limit function $u$ we also write $u=\operatorname{epi-\operatorname {lim}_{k\rightarrow +\infty }} u_{k}$ and $u_{k} \stackrel{e p i}{\longrightarrow} u$.

Remark 3.1. Another name for epi-convergence is $\Gamma$-convergence.

Epi-convergence is strongly connected to Hausdorff convergence of sublevel sets. In the following result (see [15, Lemma 5] and [6, Theorem 3.1]) we say that $\left\{u_{k} \leq t\right\} \rightarrow \emptyset$ as $k \rightarrow+\infty$ if there exists $k_{0} \in \mathbb{N}$ such that $\left\{u_{k} \leq t\right\}=\emptyset$ for all $k \geq k_{0}$.

Lemma 3.2. Let $u_{k}, u \in \operatorname{Conv}\left(\mathbb{R}^{n}\right)$. If $u_{k} \stackrel{\text { epi }}{\longrightarrow} u$, then $\left\{u_{k} \leq t\right\} \rightarrow\{u \leq t\}$ as $k \rightarrow+\infty$ for every $t \in \mathbb{R}$ with $t \neq \min _{x \in \mathbb{R}^{n}} u(x)$. Furthermore, if for every $t \in \mathbb{R}^{n}$ there exists a sequence $t_{k} \rightarrow t$ such that $\left\{u_{k} \leq t_{k}\right\} \rightarrow\{u \leq t\}$, then $u_{k} \stackrel{e p i}{\longrightarrow} u$. 
Another consequence of epi-convergence is due to [42, Theorem 7.17]

Theorem 3.3. Let $u_{k}, u: \mathbb{R}^{n} \rightarrow(-\infty,+\infty]$ be convex functions. If $u_{k}$ epi-converges to $u$, then $u$ is convex. Moreover, if $\operatorname{dom} u$ has non-empty interior, then $u_{k}$ converges uniformly to $u$ on every compact set that does not contain a boundary point of dom $u$.

Remark 3.4. By Theorem 3.3. epi-convergence on $\operatorname{Conv}\left(\mathbb{R}^{n}, \mathbb{R}\right)$ is equivalent to local uniform convergence and compact convergence. Furthermore, by [16, Example 5.13], epi-convergence on this function space is also equivalent to pointwise convergence.

A consequence of coerciveness is the so called cone property, which was established in [10, Lemma 2.5].

Lemma 3.5. For $u \in \operatorname{Conv}\left(\mathbb{R}^{n}\right)$ there exist constants $a, b \in \mathbb{R}$ with $a>0$ such that

$$
u(x)>a|x|+b
$$

for every $x \in \mathbb{R}^{n}$.

Furthermore, a uniform cone property was established in [15, Lemma 8].

Lemma 3.6. For $u_{k}, u \in \operatorname{Conv}\left(\mathbb{R}^{n}\right)$ such that $u_{k} \stackrel{e p i}{\longrightarrow} u$, there exist constants $a, b \in \mathbb{R}$ with $a>0$ such that

$$
u_{k}(x)>a|x|+b \quad \text { and } \quad u(x)>a|x|+b
$$

for every $k \in \mathbb{N}$ and $x \in \mathbb{R}^{n}$.

Recall, that for a convex function $u: \mathbb{R}^{n} \rightarrow[-\infty,+\infty]$, the convex conjugate $u^{*}$ is defined by

$$
u^{*}(x)=\sup _{y \in \mathbb{R}^{n}}(x \cdot y-u(y)),
$$

for every $x \in \mathbb{R}^{n}$. The next result can be found in [43, Theorem 1.6.13].

Lemma 3.7. If $u: \mathbb{R}^{n} \rightarrow(-\infty,+\infty]$ is a proper, lower semicontinuous, convex function, then also $u^{*}$ is a proper, lower semicontinuous, convex function and $u^{* *}=u$.

In the following we say that a function $u: \mathbb{R}^{n} \rightarrow(-\infty,+\infty]$ is super-coercive if

$$
\lim _{|x| \rightarrow+\infty} \frac{u(x)}{|x|}=+\infty \text {. }
$$

Furthermore, let int $A$ denote the interior of a set $A \subset \mathbb{R}^{n}$. As the next result shows, certain properties of a convex function correspond to certain other properties of its conjugate function, see for example [42, Theorem 11.8].

Lemma 3.8. For a proper, lower semicontinuous, convex function $u: \mathbb{R}^{n} \rightarrow(-\infty,+\infty]$, the following hold true:

- $u$ is coercive if and only if $0 \in \operatorname{int} \operatorname{dom} u^{*}$.

- $u$ is super-coercive if and only if $\operatorname{dom} u^{*}=\mathbb{R}^{n}$. 
Convex conjugation is also compatible with the valuation property, see for example [14, Lemma 3.4, Proposition 3.5]

Lemma 3.9. Let $u, v$ be proper, lower semicontinuous, convex functions. If $u \wedge v$ is convex, then so is $u^{*} \wedge v^{*}$. Furthermore,

$$
(u \wedge v)^{*}=u^{*} \vee v^{*} \quad \text { and } \quad(u \vee v)^{*}=u^{*} \wedge v^{*}
$$

The next result, which is due to Wijsman, shows that convex conjugation is a continuous operation (see [42, Theorem 11.34]).

Theorem 3.10. If $u_{k}, u: \mathbb{R}^{n} \rightarrow(-\infty,+\infty]$ are closed, proper and convex, then

$$
u_{k} \stackrel{e p i}{\longrightarrow} u \quad \text { if and only if } \quad u_{k}^{*} \stackrel{e p i}{\longrightarrow} u^{*} .
$$

For a convex, lower semicontinuous function $u: \mathbb{R}^{n} \rightarrow(-\infty,+\infty]$ and $x \in \mathbb{R}^{n}$, a vector $y \in \mathbb{R}^{n}$ is said to be a subgradient of $u$ at $x$ if

$$
u(z) \geq u(x)+(z-x) \cdot y,
$$

for all $z \in \mathbb{R}^{n}$. The (possibly empty) set of all subgradients at $x$ is called the subdifferential of $u$ at $x$ and denoted by $\partial u(x)$. In particular, if $u$ is differentiable at $x$, then $\partial u(x)=\{\nabla u(x)\}$. The next result uses subdifferentials to establish a connection between a convex function and its conjugate (see, for example, [41, Theorem 23.5]).

Lemma 3.11. For a proper, lower semicontinuous, convex function $u: \mathbb{R}^{n} \rightarrow(-\infty,+\infty]$ and $x, y \in \mathbb{R}^{n}$, the following are equivalent:

- $y \in \partial u(x)$,

- $x \in \partial u^{*}(y)$,

- $x \cdot y=u(x)+u^{*}(y)$,

- $x \in \operatorname{argmax}_{z \in \mathbb{R}^{n}}(y \cdot z-u(z))$,

- $y \in \operatorname{argmax}_{z \in \mathbb{R}^{n}}\left(x \cdot z-u^{*}(z)\right)$.

Here, $\operatorname{argmax}_{z \in V} f(z)$ denotes the points in the set $V$ at which the function values of $f$ are maximized on $V$.

For $K \in \mathcal{K}^{n}$ we consider the convex indicator function $I_{K} \in \operatorname{Conv}\left(\mathbb{R}^{n}\right)$, which is defined as

$$
I_{K}(x)= \begin{cases}0, & x \in K \\ +\infty, & x \notin K .\end{cases}
$$

Furthermore, for $K \in \mathcal{K}_{o}^{n}$ we will consider the cone function $\ell_{K} \in \operatorname{Conv}\left(\mathbb{R}^{n}\right)$, which is defined via

$$
\text { epi } \ell_{K}=\operatorname{pos}(K \times\{1\})
$$


where pos denotes the positive hull or equivalently

$$
\left\{\ell_{K} \leq t\right\}=t
$$

for every $t \geq 0$. Note, that if $K \in \mathcal{K}_{(o)}^{n}$, then $\ell_{K} \in \operatorname{Conv}\left(\mathbb{R}^{n}, \mathbb{R}\right)$. Furthermore, for every $K \in \mathcal{K}_{(o)}^{n}$ we have $\ell_{K}=h\left(K^{*}, \cdot\right)$ where $h\left(K^{*}, \cdot\right)$ denotes the support function of $K^{*}$. For convex bodies $L \in \mathcal{K}^{n}$ the support function can be defined as $h(L, x)=\max _{y \in L} y \cdot x$ for every $x \in \mathbb{R}^{n}$.

The following relations for $u \in \operatorname{Conv}\left(\mathbb{R}^{n}\right), K \in \mathcal{K}_{o}^{n}, L \in \mathcal{K}^{n}, t \in \mathbb{R}, x \in \mathbb{R}^{n}$, translations $\tau_{y}(x)=x+y$ and $\phi \in \mathrm{SL}(n)$ are easy to see:

$$
\begin{aligned}
(u+t)^{*}(x) & =u^{*}(x)-t \\
\left(\ell_{K}\right)^{*}(x) & =\mathrm{I}_{K^{*}}(x) \\
\mathrm{I}_{L}^{*}(x) & =h(L, x) \\
\left(u \circ \tau_{y}^{-1}\right)^{*}(x) & =u^{*}(x)+y \cdot x \\
\left(u \circ \phi^{-1}\right)^{*}(x) & =\left(u^{*} \circ \phi^{t}\right)(x)
\end{aligned}
$$

Next, for $\delta>0$ and $u \in \operatorname{Conv}\left(\mathbb{R}^{n}\right)$ we define the regularization $\operatorname{reg}_{\delta} u$ as

$$
\operatorname{reg}_{\delta} u=\left(u^{*}+\mathrm{I}_{\delta^{-1} Q^{n}}\right)^{*} .
$$

Lemma 3.12. For $u, u_{j}, v \in \operatorname{Conv}\left(\mathbb{R}^{n}\right)$ with $u \wedge v \in \operatorname{Conv}\left(\mathbb{R}^{n}\right), K \in \mathcal{K}_{o}^{n}$ and $\delta>0$ we have the following properties.

1. $\operatorname{reg}_{\delta} u \in \operatorname{Conv}\left(\mathbb{R}^{n}, \mathbb{R}\right)$.

2. $\operatorname{reg}_{\delta}(u \wedge v)=\operatorname{reg}_{\delta} u \wedge \operatorname{reg}_{\delta} v$ and $\operatorname{reg}_{\delta}(u \vee v)=\operatorname{reg}_{\delta} u \vee \operatorname{reg}_{\delta} v$.

3. $\operatorname{reg}_{\delta} u \stackrel{e p i}{\longrightarrow} u$ as $\delta \rightarrow 0$.

4. If $u_{j} \rightarrow u$, then $\operatorname{reg}_{\delta} u_{j} \stackrel{e p i}{\longrightarrow} \operatorname{reg}_{\delta} u$ for sufficiently small $\delta>0$.

5. $\operatorname{reg}_{\delta}(u+t)=\left(\operatorname{reg}_{\delta} u\right)+t$ for every $t \in \mathbb{R}$.

6. $\operatorname{reg}_{\delta}\left(u \circ \tau^{-1}\right)=\left(\operatorname{reg}_{\delta} u\right) \circ \tau^{-1}$ for every translation $\tau$ on $\mathbb{R}^{n}$.

7. There exists a unique $K_{\delta} \in \mathcal{K}_{(o)}^{n}$ such that $\operatorname{reg}_{\delta} \ell_{K}=\ell_{K_{\delta}}$. In particular, $K_{\delta}=\operatorname{conv}(K \cup$ $\left.\delta C^{n}\right)$. Furthermore, $K \subseteq K_{\delta}$ with equality iff $\delta C^{n} \subseteq K$. In particular, $K_{\delta} \rightarrow K$ in the Hausdorff metric as $\delta \rightarrow 0$.

Proof. The proofs of 1, 2, 3 and 4 follow along similar lines as the proofs of corresponding results for the well-known Lipschitz regularization, see for example [14, Section 4]. Since for any proper, lower semicontinuous, convex function $u$ and $t \in \mathbb{R}$ we have $(u+t)^{*}=u^{*}-t$, it follows for $u \in \operatorname{Conv}\left(\mathbb{R}^{n}\right)$ and $\delta>0$ that

$$
\operatorname{reg}_{\delta}(u+t)=\left(u^{*}-t+\mathrm{I}_{\delta^{-1} Q^{n}}\right)^{*}=\left(u^{*}+\mathrm{I}_{\delta^{-1} Q^{n}}\right)^{*}+t=\left(\operatorname{reg}_{\delta} u\right)+t .
$$

Similarly, one shows covariance with respect to translations. 
Next, let $K \in \mathcal{K}_{o}^{n}$ and observe that

$$
\operatorname{reg}_{\delta} \ell_{K}=\left(\mathrm{I}_{K^{*}}+\mathrm{I}_{\delta^{-1} Q^{n}}\right)^{*}=\left(\mathrm{I}_{K^{*} \cap \delta^{-1} Q^{n}}\right)^{*}=\ell_{\left(K^{*} \cap \delta^{-1} Q^{n}\right)^{*}}
$$

Since $K^{*} \cap \delta^{-1} Q^{n}$ is an element of $\mathcal{K}_{(o)}^{n}$, we have $\operatorname{reg}_{\delta} \ell_{K}=\ell_{K_{\delta}}$ with

$$
K_{\delta}:=\left(K^{*} \cap \delta^{-1} Q^{n}\right)^{*}=\operatorname{conv}\left(K \cup \delta C^{n}\right) \in \mathcal{K}_{(o)}^{n},
$$

see for example [43, Theorem 1.6.3]. Furthermore, since polarity is order reversing, it is easy to see that $K \subseteq K_{\delta}$. Finally, $\delta C^{n} \subseteq K$ is equivalent to $K=K_{\delta}$.

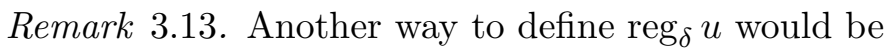

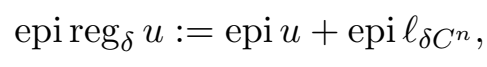

where + denotes the Minkowski addition (that is adding each element of one set with each element of the other set). For details see [43, Section 1.6].

To proof Lemma 5.1 we will also need another class of convex functions. We call a function $u \in \operatorname{Conv}\left(\mathbb{R}^{n}\right)$ piecewise affine, if there exist finitely many $n$-dimensional convex polyhedra $Q_{1}, \ldots, Q_{m}$ with pairwise disjoint interiors such that $\bigcup_{i=1}^{m} Q_{i}=\mathbb{R}^{n}$ and the restriction of $u$ to each $Q_{i}$ is an affine function. We will denote the set of piecewise affine convex functions by $\operatorname{Conv}_{\text {p.a. }}\left(\mathbb{R}^{n}\right)$.

Since epi-convergence on $\operatorname{Conv}\left(\mathbb{R}^{n}, \mathbb{R}\right)$ is equivalent to pointwise convergence, the following result is easy to see (see also [15, Lemma 11]).

Lemma 3.14. $\operatorname{Conv}_{\text {p.a. }}\left(\mathbb{R}^{n}\right)$ is a dense subset of $\operatorname{Conv}\left(\mathbb{R}^{n}, \mathbb{R}\right)$.

\section{Valuations on Convex Functions}

In this section we will consider several $\mathrm{SL}(n)$ invariant valuations on spaces of convex functions, most of which can be interpreted as functional analogs of well known operators on $\mathcal{K}^{n}$.

In the following we say that a real-valued valuation $\mathrm{Z}$, defined on a subset $\mathcal{S}$ of $\operatorname{Conv}\left(\mathbb{R}^{n}\right)$, is homogeneous of degree $p \in \mathbb{R}$ if $\mathrm{Z}\left(u_{\lambda}\right)=\lambda^{p} \mathrm{Z}(u)$ for every $u \in \mathcal{S}$ and $\lambda>0$, where $u_{\lambda}(x):=u\left(\frac{x}{\lambda}\right)$. Note, that $\left(u_{\lambda}\right)^{*}=\left(u^{*}\right)_{\lambda^{-1}}$.

\subsection{Minimum and Integral}

The following operator can be seen as a functional analog of the Euler characteristic.

Lemma 4.1 ([15], Lemma 12). For a continuous function $\zeta: \mathbb{R} \rightarrow \mathbb{R}$ the map

$$
u \mapsto \zeta\left(\min _{x \in \mathbb{R}^{n}} u(x)\right)
$$

defines a continuous, $\mathrm{SL}(n)$ and translation invariant valuation on $\operatorname{Conv}\left(\mathbb{R}^{n}\right)$ that is homogeneous of degree 0. 
The properties of the next operator are similar to those of the volume operator of convex bodies.

Lemma 4.2 ([15], Lemma 16). For a continuous function $\zeta: \mathbb{R} \rightarrow[0, \infty)$ with finite moment of order $n-1$, the map

$$
u \mapsto \int_{\operatorname{dom} u} \zeta(u(x)) \mathrm{d} x
$$

defines a non-negative, continuous, $\operatorname{SL}(n)$ and translation invariant valuation on $\operatorname{Conv}\left(\mathbb{R}^{n}\right)$ that is homogeneous of degree $n$.

\subsection{Polar Volumes}

By Lemma 3.8 it is easy to see that

$$
\begin{aligned}
\operatorname{Conv}\left(\mathbb{R}^{n}\right)^{*}: & =\left\{u^{*}: u \in \operatorname{Conv}\left(\mathbb{R}^{n}\right)\right\} \\
& =\left\{u: \mathbb{R}^{n} \rightarrow(-\infty, \infty]: u \text { is proper, l.s.c., convex, } 0 \in \operatorname{int} \operatorname{dom} u\right\} .
\end{aligned}
$$

Now for a valuation $\mathrm{Z}$ on $\operatorname{Conv}\left(\mathbb{R}^{n}\right)$, the map

$$
u \mapsto \mathrm{Z}^{*}(u)=\mathrm{Z}\left(u^{*}\right)
$$

defines a valuation on $\operatorname{Conv}\left(\mathbb{R}^{n}\right)^{*}$ since by Lemma 3.9

$$
\begin{aligned}
\mathrm{Z}^{*}(u \wedge v)+\mathrm{Z}^{*}(u \vee v) & =\mathrm{Z}\left((u \wedge v)^{*}\right)+\mathrm{Z}\left((u \vee v)^{*}\right) \\
& =\mathrm{Z}\left(u^{*} \vee v^{*}\right)+\mathrm{Z}\left(u^{*} \wedge v^{*}\right) \\
& =\mathrm{Z}\left(u^{*}\right)+\mathrm{Z}\left(v^{*}\right) \\
& =\mathrm{Z}^{*}(u)+\mathrm{Z}^{*}(v)
\end{aligned}
$$

for every $u, v \in \operatorname{Conv}\left(\mathbb{R}^{n}\right)^{*}$ such that $u \wedge v \in \operatorname{Conv}\left(\mathbb{R}^{n}\right)^{*}$. Furthermore, if Z is continuous and $\mathrm{SL}(n)$ invariant, then by Theorem 3.10 and (6) the operator $\mathrm{Z}^{*}$ is also continuous and $\mathrm{SL}(n)$ invariant. Moreover, if $\mathrm{Z}$ is translation invariant, then $\mathrm{Z}^{*}$ is linear invariant, that is

$$
\mathrm{Z}^{*}(u+l)=\mathrm{Z}^{*}(u)
$$

for every $u \in \operatorname{Conv}\left(\mathbb{R}^{n}\right)^{*}$ and $l: \mathbb{R}^{n} \rightarrow \mathbb{R}^{n}$ of the form $l(x)=y \cdot x$ with some $y \in \mathbb{R}^{n}$. Lastly, if $Z$ is homogeneous of degree $p \in \mathbb{R}$, then

$$
\mathrm{Z}^{*}\left(u_{\lambda}\right)=\mathrm{Z}\left(\left(u_{\lambda}\right)^{*}\right)=\mathrm{Z}\left(\left(u^{*}\right)_{\lambda^{-1}}\right)=\lambda^{-p} \mathrm{Z}\left(u^{*}\right)=\lambda^{-p} \mathrm{Z}^{*}(u),
$$

which shows that $\mathrm{Z}^{*}$ is homogeneous of degree $-p$. Hence, by Lemma 4.2 we have the following result.

Lemma 4.3. For a continuous function $\zeta: \mathbb{R} \rightarrow[0, \infty)$ with finite moment of order $n-1$, the map

$$
u \mapsto \int_{\operatorname{dom} u^{*}} \zeta\left(u^{*}(x)\right) \mathrm{d} x
$$

defines a non-negative, continuous, $\mathrm{SL}(n)$ and linear invariant valuation on $\operatorname{Conv}\left(\mathbb{R}^{n}\right)^{*}$ that is homogeneous of degree $-n$. 
Remark 4.4. If (8) is evaluated for functions of the type $\ell_{K}$ or $\mathrm{I}_{K}$ with $K \in \mathcal{K}_{(o)}^{n}$, a multiple of $V_{n}^{*}(K)$ is obtained. However, if we evaluate for the support function $h(K, \cdot)$ of a convex body $K \in \mathcal{K}^{n}$, we obtain

$$
\int_{\operatorname{dom} h^{*}(K, \cdot)} \zeta\left(h^{*}(K, x)\right) \mathrm{d} x=\int_{K} \zeta\left(\mathrm{I}_{K}(x)\right) \mathrm{d} x=\zeta(0) V_{n}(K) .
$$

Note, that for every $y \in \mathbb{R}^{n}$ we have $h(K+y, x)=h(K, x)+y \cdot x$ for $x \in \mathbb{R}^{n}$. In particular, the valuation defined in (8) is not translation invariant anymore.

In order to obtain a translation invariant analog of the polar volume on $\operatorname{Conv}\left(\mathbb{R}^{n}, \mathbb{R}\right)$ we need the following result.

Proposition 4.5 ([14], Section 10.4 and Theorem 11.1). For a continuous function $\zeta: \mathbb{R} \times \mathbb{R}^{n} \rightarrow$ $\mathbb{R}$ with compact support, the map

$$
u \mapsto \int_{\operatorname{dom} u^{*}} \zeta\left(\nabla u^{*}(x) \cdot x-u^{*}(x), x\right) \mathrm{d} x
$$

defines a continuous and translation invariant valuation on $\operatorname{Conv}\left(\mathbb{R}^{n}\right)$.

Lemma 4.6. For a continuous function $\zeta: \mathbb{R} \rightarrow \mathbb{R}$ such that $\zeta(t)=0$ for all $t \geq T$ with some $T \in \mathbb{R}$, the map

$$
u \mapsto \int_{\operatorname{dom} u^{*}} \zeta\left(\nabla u^{*}(x) \cdot x-u^{*}(x)\right) \mathrm{d} x
$$

defines a continuous, $\mathrm{SL}(n)$ and translation invariant valuation on $\operatorname{Conv}\left(\mathbb{R}^{n}, \mathbb{R}\right)$ that is homogeneous of degree $-n$.

Proof. Let $u_{k}, u \in \operatorname{Conv}\left(\mathbb{R}^{n}, \mathbb{R}\right)$ such that $u_{k} \stackrel{e p i}{\longrightarrow} u$. By Lemma 3.6, there exist $b \in \mathbb{R}$ and $R>0$ such that $u_{k}(x), u(x) \geq b$ for every $x \in \mathbb{R}^{n}$ and $u_{k}(x), u(x) \geq T$ for every $x \in \mathbb{R}^{n}$ with $|x| \geq R$ and every $k \in \mathbb{N}$. By Theorem 3.3 the functions $u_{k}$ converge uniformly to $u$ on $B_{R}^{n}:=R \cdot B^{n}$. Furthermore, as convex functions they are Lipschitz continuous on this set and therefore there exists $C>0$ such that $|y| \leq C$ for every $y \in \partial u_{k}(x) \cup \partial u(x)$ with $x \in B_{R}^{n}$ and $k \in \mathbb{N}$. Hence, $u(x), u_{k}(x) \geq T$ for every $x \in \mathbb{R}^{n}$ such that there exists $y \in \partial u(x) \cup u_{k}(x)$ with $|y|>C$. Lemma 3.11 now shows that for every pair $x, y \in \mathbb{R}^{n}$ such that $y \in \partial u(x)$ and $|y|>C$ we have

$$
x \cdot y-u^{*}(y)=u(x) \geq T
$$

and similarly if $y \in \partial u_{k}(x)$ with $|y|>C$

$$
x \cdot y-u_{k}^{*}(y)=u_{k}(x) \geq T,
$$

for every $k \in \mathbb{N}$. Using Lemma 3.11 again and considering that convex functions are differentiable a.e. this gives

$$
\nabla u^{*}(y) \cdot y-u^{*}(y) \geq T \quad \text { and } \quad \nabla u_{k}^{*}(y) \cdot y-u_{k}^{*}(y) \geq T
$$

for a.e. $y \in \mathbb{R}^{n}$ with $|y|>C$ and for every $k \in \mathbb{N}$.

Next, let $\xi: \mathbb{R}^{n} \rightarrow \mathbb{R}$ be a smooth function with compact support such that $\xi \equiv 1$ on $B_{C}^{n}$ and let $\eta: \mathbb{R} \rightarrow \mathbb{R}$ be a smooth function with compact support such that $\eta \equiv 1$ on $[b, T]$. Define 
$\bar{\zeta} \in C\left(\mathbb{R} \times \mathbb{R}^{n}\right)$ as $\bar{\zeta}(t, y):=\zeta(t, y) \eta(t) \xi(y)$ for every $t \in \mathbb{R}$ and $y \in \mathbb{R}^{n}$. Using Proposition 4.5 we now have

$$
\begin{aligned}
\int_{\operatorname{dom} u_{k}^{*}} \zeta\left(\nabla u_{k}^{*}(x) \cdot x-u_{k}^{*}(x)\right) \mathrm{d} x=\int_{\operatorname{dom} u_{k}^{*}} \bar{\zeta}\left(\nabla u_{k}^{*}(x) \cdot x-u_{k}^{*}(x), x\right) \mathrm{d} x \\
\stackrel{k \rightarrow \infty}{\longrightarrow} \int_{\operatorname{dom} u^{*}} \bar{\zeta}\left(\nabla u^{*}(x) \cdot x-u^{*}(x), x\right) \mathrm{d} x=\int_{\operatorname{dom} u^{*}} \zeta\left(\nabla u^{*}(x) \cdot x-u^{*}(x)\right) \mathrm{d} x,
\end{aligned}
$$

which shows that (9) defines a continuous, translation invariant valuation on $\operatorname{Conv}\left(\mathbb{R}^{n}, \mathbb{R}\right)$.

It remains to show $\mathrm{SL}(n)$ invariance and homogeneity. Therefore, let $\phi \in \mathrm{SL}(n), \lambda>0$ and observe that

$$
\begin{aligned}
\int_{\operatorname{dom}\left(u_{\lambda} \circ \phi^{-1}\right)^{*}} \zeta\left(\nabla\left(u_{\lambda} \circ \phi^{-1}\right)^{*}(x)\right. & \left.\cdot x-\left(u_{\lambda} \circ \phi^{-1}\right)^{*}(x)\right) \mathrm{d} x \\
& =\int_{\operatorname{dom} u^{*} \circ \lambda \phi^{t}} \zeta\left(\nabla\left(u^{*} \circ \lambda \phi^{t}\right)(x) \cdot x-u^{*}\left(\lambda \phi^{t} x\right)\right) \mathrm{d} x \\
& =\int_{\left(\lambda \phi^{t}\right)^{-1} \operatorname{dom} u^{*}} \zeta\left(\nabla u^{*}\left(\lambda \phi^{t} x\right) \cdot \lambda \phi^{t} x-u^{*}\left(\lambda \phi^{t} x\right)\right) \mathrm{d} x \\
& =\lambda^{-n} \int_{\operatorname{dom} u^{*}} \zeta\left(\nabla u^{*}(x) \cdot x-u^{*}(x)\right) \mathrm{d} x,
\end{aligned}
$$

which concludes the proof.

Remark 4.7. Lemma 4.6 shows that there exist non-trivial, continuous and translation invariant valuations on $\operatorname{Conv}\left(\mathbb{R}^{n}, \mathbb{R}\right)$ that are homogeneous of degree $-n$. Hence, a direct analog of Theorem 2.1 for valuations on $\operatorname{Conv}\left(\mathbb{R}^{n}, \mathbb{R}\right)$ is not true.

Remark 4.8. It is easy to see that (9) does not extend to $\operatorname{Conv}\left(\mathbb{R}^{n}\right)$. In order to see that, let $u=\mathrm{I}_{K}+t$ with $K \in \mathcal{K}_{(o)}^{n}$ and $t \in \mathbb{R}$ such that $\zeta(t) \neq 0$. We have $u^{*}=\ell_{K^{*}}-t$ and furthermore $\nabla \ell_{K^{*}}(x) \cdot x=\ell_{K^{*}}(x)$ for a.e. $x \in \mathbb{R}^{n}$. Thus,

$$
\int_{\operatorname{dom} u^{*}} \zeta\left(\nabla u^{*}(x) \cdot x-u^{*}(x)\right) \mathrm{d} x=\int_{\mathbb{R}^{n}} \zeta(t) \mathrm{d} x
$$

which is not finite.

\subsection{Further SL $(n)$ Invariant Valuations}

We briefly discuss further $\mathrm{SL}(n)$ invariant valuations on spaces of convex functions. It is easy to see that $K \mapsto V_{0}\left(K^{*}\right)$ defines an $\mathrm{SL}(n)$ invariant, continuous valuation on $\mathcal{K}_{(o)}^{n}$. Obviously, in this case $V_{0}\left(K^{*}\right)=1=V_{0}(K)$ and such an operator does not explicitly appear in Theorem 1.3. For a convex function $u$ on $\mathbb{R}^{n}$ however, the values $\zeta\left(\min _{x \in \mathbb{R}^{n}} u(x)\right)$ and $\zeta\left(\min _{x \in \mathbb{R}^{n}} u^{*}(x)\right)$ do not coincide in general. Note, that

$$
u^{*}(0)=\sup _{y \in \mathbb{R}^{n}}(0-u(y))=-\inf _{y \in \mathbb{R}^{n}} u(y),
$$

and similarly $u(0)=u^{* *}(0)=-\inf _{y \in \mathbb{R}^{n}} u^{*}(y)$. Hence, $u^{*}$ is bounded from below and attains its minimum if and only if $0 \in \operatorname{dom} u$. Thus, using Theorem 3.10 we have the following result dual to Lemma 4.1 
Lemma 4.9. For a continuous function $\zeta: \mathbb{R} \rightarrow \mathbb{R}$ the map

$$
u \mapsto \zeta\left(\min _{x \in \mathbb{R}^{n}} u^{*}(x)\right)=\zeta(-u(0))
$$

defines a continuous, $\mathrm{SL}(n)$ and linear invariant valuation on

$$
\left\{u: \mathbb{R}^{n} \rightarrow(-\infty, \infty]: u \text { is proper, l.s.c., convex }, 0 \in \operatorname{dom} u\right\}
$$

that is homogeneous of degree 0.

If one removes the assumption of translation invariance in Theorem 1.1, the additional valuation

$$
K \mapsto V_{n}(\operatorname{conv}\{0, K\})
$$

appears, see for example [35, Corollary 2.3]. As a functional analog of $\operatorname{conv}\{0, K\}$ on $\operatorname{Conv}\left(\mathbb{R}^{n}\right)$ we associate with a function $u \in \operatorname{Conv}\left(\mathbb{R}^{n}\right)$ the function $u_{0} \in \operatorname{Conv}\left(\mathbb{R}^{n}\right)$ which is defined via

$$
u_{0}:=\left(u^{*} \vee 0\right)^{*} .
$$

It is not hard to see that this can also be written as

$$
\text { epi } u_{0}=\operatorname{conv}\{0 \times[0, \infty) \text {, epi } u\}
$$

Lemma 4.10. For a continuous function $\zeta: \mathbb{R} \rightarrow[0, \infty)$ with finite moment of order $n-1$, the map

$$
u \mapsto \int_{\operatorname{dom} u_{0}} \zeta\left(u_{0}(x)\right) \mathrm{d} x
$$

defines a non-negative, continuous and $\mathrm{SL}(n)$ invariant valuation on $\operatorname{Conv}\left(\mathbb{R}^{n}\right)$.

Proof. Note, that by Lemma 3.8 the function $u_{0}$ is coercive if and only if $0 \in \operatorname{int} \operatorname{dom} u_{0}^{*}$, which is true since $u$ is coercive and therefore $0 \in \operatorname{int} \operatorname{dom} u^{*}$. Hence, by Lemma 4.2 the map (10) is well defined. Furthermore, by Theorem 3.10 and (6) it is easy to see that this operator is continuous and $\operatorname{SL}(n)$ invariant. It remains to show the valuation property. Therefore, let $u, v \in \operatorname{Conv}\left(\mathbb{R}^{n}\right)$ such that $u \wedge v \in \operatorname{Conv}\left(\mathbb{R}^{n}\right)$. Since

$$
\begin{aligned}
& \left(u^{*} \vee 0\right) \vee\left(v^{*} \vee 0\right)=\left(u^{*} \vee v^{*}\right) \vee 0 \\
& \left(u^{*} \vee 0\right) \wedge\left(v^{*} \vee 0\right)=\left(u^{*} \wedge v^{*}\right) \vee 0
\end{aligned}
$$

it follows from Lemma 3.9 and Lemma 4.2 that (10) defines a valuation.

Similar to Lemma 4.3 we immediately obtain the following result.

Lemma 4.11. For a continuous function $\zeta: \mathbb{R} \rightarrow[0, \infty)$ with finite moment of order $n-1$, the map

$$
u \mapsto \int_{\operatorname{dom}\left(u^{*}\right)_{0}} \zeta\left(\left(u^{*}\right)_{0}(x)\right) \mathrm{d} x
$$

defines a non-negative, continuous and $\mathrm{SL}(n)$ invariant valuation on $\operatorname{Conv}\left(\mathbb{R}^{n}\right)^{*}$. 
We want to close this section with a result dual to Lemma 4.6. Note, that the space dual to $\operatorname{Conv}\left(\mathbb{R}^{n}, \mathbb{R}\right)$ is

$$
\begin{aligned}
\operatorname{Conv}\left(\mathbb{R}^{n}, \mathbb{R}\right)^{*} & =\left\{u^{*}: u \in \operatorname{Conv}\left(\mathbb{R}^{n}, \mathbb{R}\right)\right\} \\
& =\left\{u: \mathbb{R}^{n} \rightarrow(-\infty, \infty]: u \text { is proper, l.s.c., convex, super-coercive, } 0 \in \operatorname{int} \operatorname{dom} u\right\} .
\end{aligned}
$$

Lemma 4.12. For a continuous function $\zeta: \mathbb{R} \rightarrow \mathbb{R}$ such that $\zeta(t)=0$ for all $t \geq T$ with some $T \in \mathbb{R}$, the map

$$
u \mapsto \int_{\operatorname{dom} u} \zeta(\nabla u(x) \cdot x-u(x)) \mathrm{d} x
$$

defines a continuous, $\mathrm{SL}(n)$ and linear invariant valuation on $\operatorname{Conv}\left(\mathbb{R}^{n}, \mathbb{R}\right)^{*}$ that is homogeneous of degree $n$.

\section{Classification of Valuations}

\subsection{General Considerations}

The following result is a variant of [15, Lemma 17] and is based on a principle that was introduced in [32, Lemma 8]. As there was an error in the induction step of the original proof, we give a new corrected proof here. Furthermore, the author is most grateful to Jin Li for pointing out the mistake.

Lemma 5.1. Let $\langle A,+\rangle$ be a topological abelian semigroup with cancellation law and let $\mathrm{Z}_{1}, \mathrm{Z}_{2}$ : $\operatorname{Conv}\left(\mathbb{R}^{n}, \mathbb{R}\right) \rightarrow\langle A,+\rangle$ be continuous, translation invariant valuations. If $\mathrm{Z}_{1}\left(\ell_{P}+t\right)=\mathrm{Z}_{2}\left(\ell_{P}+t\right)$ for every $P \in \mathcal{P}_{(o)}^{n}$ and $t \in \mathbb{R}$, then $\mathrm{Z}_{1} \equiv \mathrm{Z}_{2}$ on $\operatorname{Conv}\left(\mathbb{R}^{n}, \mathbb{R}\right)$.

Proof. By Lemma 3.14 and the continuity of $\mathrm{Z}_{1}$ and $\mathrm{Z}_{2}$, it is enough to show that $\mathrm{Z}_{1}$ and $\mathrm{Z}_{2}$ coincide on $\operatorname{Conv}_{\text {p.a. }}\left(\mathbb{R}^{n}\right)$. Hence, w.l.o.g. let $u \in \operatorname{Conv}_{\text {p.a. }}\left(\mathbb{R}^{n}\right)$ and set $U=\operatorname{epi} u \subset \mathbb{R}^{n} \times \mathbb{R}$. Since $u$ does not attain the value $+\infty$, none of the facet hyperplanes of $U$ (i.e. the hyperplanes in $\mathbb{R}^{n+1}$ that have an $n$-dimensional intersection with the boundary of $U$ ) is parallel to the $x_{n+1}$-axis. We call $U$ singular if $U$ has $n$ facet hyperplanes whose intersection contains a line parallel to the coordinate hyperplane $\left\{x_{n+1}=0\right\}$. By continuity it is enough to restrict to the cases where $U$ is not singular.

We will use induction on the number $m$ of vertices of $U$. If $m=1$, then $U$ has just one vertex $p_{0}=\left(x_{0}, t_{0}\right)$ with $x_{0} \in \mathbb{R}^{n}$ and $t_{0} \in \mathbb{R}$ and therefore $U$ is the translate of a polyhedral cone. Denote by $Q \in \mathcal{P}^{n}$ the projection onto the first $n$ coordinates of $U \cap\left\{x_{n+1}=t_{0}+1\right\}$ and let $P=Q-x_{0}$. Since none of the facet hyperplanes of $U$ is parallel to the $x_{n+1}$-axis and $U$ is polyhedral, the set $P$ is a polytope that contains the origin in its interior and $u$ is a translate of $\ell_{P}+t_{0}$. As $\mathrm{Z}_{1}$ and $\mathrm{Z}_{2}$ are translation invariant, we have $\mathrm{Z}_{1}(u)=\mathrm{Z}_{2}(u)$.

Now let $U$ have $m>1$ vertices and assume that $\mathrm{Z}_{1}$ and $\mathrm{Z}_{2}$ coincide on every piecewise affine convex function whose epigraph has most $m-1$ vertices. Since $U$ is not singular, there exists a unique vertex $p_{0}=\left(x_{0}, t_{0}\right)$ of $U$ with $x_{0} \in \mathbb{R}^{n}$ and $t_{0} \in \mathbb{R}$ such that $t_{0}$ is minimal. Let $H_{1}, \ldots, H_{j}$ be the facet hyperplanes of $U$ that contain $p_{0}$ and define $U_{0}$ as the intersection of the corresponding half-spaces. By the properties of $U$ and the choice of $p_{0}$ the set $U_{0}$ is the epigraph 
of a function $u_{0} \in \operatorname{Conv}_{\text {p.a. }}\left(\mathbb{R}^{n}\right)$ such that $u_{0}$ is a translate of $\ell_{P_{0}}+t_{0}$ where $P_{0} \in \mathcal{P}_{(o)}^{n}$ is such that $P_{0}+x_{0}$ is the projection onto the first $n$ coordinates of $U_{0} \cap\left\{x_{n+1}=t_{0}+1\right\}$.

Next, let $p_{1} \subset \mathbb{R}^{n+1}$ be a vertex of $U$ with second smallest $x_{n+1}$ coordinate. Since $u$ is a convex piecewise affine function there exists $\varepsilon>0$ such that $p_{1}+\operatorname{epi} \ell_{[-\varepsilon, \varepsilon]^{n}} \subset U \subset U_{0}$. Let $F_{1}, \ldots, F_{j}$ denote the facets of $U_{0}$ and set $\bar{F}_{i}=F_{i} \backslash\left(F_{i} \cap U\right)$ for $1 \leq i \leq j$. We now define the polyhedron $U_{1}$ as

$$
U_{1}=\operatorname{cl}\left(\operatorname{conv}\left\{\bar{F}_{1}, \ldots, \bar{F}_{j}, p_{1}+\operatorname{epi} \ell_{[-\varepsilon, \varepsilon]^{n}}\right\}\right) \subset U_{0},
$$

where cl denotes the closure of a set. By definition, $U_{1}$ is the epigraph of a polyhedral convex function $u_{1} \in \operatorname{Conv}\left(\mathbb{R}^{n}\right)$. Since $p_{1}+$ epi $\ell_{[-\varepsilon, \varepsilon]^{n}} \subset U_{1}$ the function $u_{1}$ does not attain the value $+\infty$ and therefore $u_{1} \in \operatorname{Conv}_{\text {p.a. }}\left(\mathbb{R}^{n}\right)$. Furthermore, $U_{1}$ and $U \cap U_{1}$ each have at most $m-1$ vertices since every vertex of $U_{1}$ is also vertex of $U$ but $p_{0}$ is not a vertex of $U_{1}$. Hence, by the induction assumption $\mathrm{Z}_{1}$ and $\mathrm{Z}_{2}$ coincide on $u_{1}$ and $u \vee u_{1}$. Moreover, since $U \cup U_{1}=U_{0}$ we have $u \wedge u_{1}=u_{0}$ and therefore by the valuation property

$$
\begin{aligned}
\mathrm{Z}_{1}(u)+\mathrm{Z}_{1}\left(u_{1}\right) & =\mathrm{Z}_{1}\left(u \vee u_{1}\right)+\mathrm{Z}_{1}\left(u \wedge u_{1}\right) \\
& =\mathrm{Z}_{1}\left(u \vee u_{1}\right)+\mathrm{Z}_{1}\left(u_{0}\right) \\
& =\mathrm{Z}_{2}\left(u \vee u_{1}\right)+\mathrm{Z}_{2}\left(u_{0}\right) \\
& =\mathrm{Z}_{2}\left(u \vee u_{1}\right)+\mathrm{Z}_{2}\left(u \wedge u_{1}\right)=\mathrm{Z}_{2}(u)+\mathrm{Z}_{2}\left(u_{1}\right),
\end{aligned}
$$

which completes the proof.

\subsection{Considerations on $\mathrm{SL}(n)$ Invariant Valuations}

In the following let $n \geq 2$.

Lemma 5.2. If $\mathrm{Z}: \operatorname{Conv}\left(\mathbb{R}^{n}, \mathbb{R}\right) \rightarrow[0,+\infty)$ is a continuous and $\mathrm{SL}(n)$ invariant valuation, then there exist continuous functions $\psi_{0}, \psi_{1}, \psi_{2}: \mathbb{R} \rightarrow[0,+\infty)$ such that

$$
\mathrm{Z}\left(\ell_{K}+t\right)=\psi_{0}(t)+\psi_{1}(t) V_{n}(K)+\psi_{2}(t) V_{n}^{*}(K)
$$

for every $K \in \mathcal{K}_{(o)}^{n}$ and $t \in \mathbb{R}$.

Proof. For $t \in \mathbb{R}$, define $\mu_{t}: \mathcal{K}_{(o)}^{n} \rightarrow \mathbb{R}$ as

$$
\mu_{t}(K)=\mathrm{Z}\left(\ell_{K}+t\right)
$$

Since for $K, L \in \mathcal{K}_{(o)}^{n}$ such that $K \cup L \in \mathcal{K}_{(o)}^{n}$ one has

$$
\ell_{K \cup L}=\ell_{K} \wedge \ell_{L} \quad \text { and } \quad \ell_{K \cap L}=\ell_{K} \vee \ell_{L}
$$

the map $\mu_{t}$ defines a valuation on $\mathcal{K}_{(o)}^{n}$ for every $t \in \mathbb{R}$. Furthermore, by (5) and Lemma 3.2 it is easy to see that $\mu_{t}$ is continuous and $\mathrm{SL}(n)$ invariant. Hence, by Theorem 1.3, there exist constants $c_{0, t}, c_{1, t}, c_{2, t} \in \mathbb{R}$ such that

$$
\mathrm{Z}\left(\ell_{K}+t\right)=\mu_{t}(K)=c_{0, t}+c_{1, t} V_{n}(K)+c_{2, t} V_{n}^{*}(K),
$$


for every $K \in \mathcal{K}_{(o)}^{n}$. This defines functions $\psi_{0}(t)=c_{0, t}, \psi_{1}(t)=c_{1, t}$ and $\psi_{2}(t)=c_{2, t}$. Fix $K \in \mathcal{K}_{(o)}^{n}$. For every $\lambda>0$ and $t \in \mathbb{R}$ we have

$$
\mathrm{Z}\left(\ell_{\lambda K}+t\right)=\psi_{0}(t)+\lambda^{n} \psi_{1}(t) V_{n}(K)+\lambda^{-n} \psi_{2}(t) V_{n}^{*}(K)
$$

Considering, that $t \mapsto \mathrm{Z}\left(\ell_{\lambda K}+t\right)$ is continuous and taking different values for $\lambda$ and linear combinations of (11), it is easy to see, that $\psi_{0}, \psi_{1}$ and $\psi_{2}$ must be continuous. For example, subtracting (11) with $\lambda=1$ from the general case gives

$$
\mathrm{Z}\left(\ell_{\lambda K}+t\right)-\mathrm{Z}\left(\ell_{K}+t\right)=\left(\lambda^{n}-1\right) \psi_{1}(t) V_{n}(K)+\left(\lambda^{-n}-1\right) \psi_{2}(t) V_{n}^{*}(K) .
$$

Taking again different values for $\lambda$ in the equation above and considering that $V_{n}(K) \neq 0$ for every $K \in \mathcal{K}_{(o)}^{n}$, one can see that $\psi_{1}$ is continuous. Similarly, one can see that $\psi_{0}$ and $\psi_{2}$ are continuous. Furthermore, by homogeneity, it is easy to see that $\psi_{0}, \psi_{1}$ and $\psi_{2}$ are non-negative.

For a continuous and $\operatorname{SL}(n)$ invariant valuation $\mathrm{Z}: \operatorname{Conv}\left(\mathbb{R}^{n}, \mathbb{R}\right) \rightarrow \mathbb{R}$, we call the functions $\psi_{0}, \psi_{1}$ and $\psi_{2}$ the growth functions of Z. By Lemma 5.1, we have the following result.

Lemma 5.3. Every continuous, $\mathrm{SL}(n)$ and translation invariant valuation $\mathrm{Z}: \operatorname{Conv}\left(\mathbb{R}^{n}, \mathbb{R}\right) \rightarrow \mathbb{R}$ is uniquely determined by its growth functions.

In order to classify valuations on $\operatorname{Conv}\left(\mathbb{R}^{n}, \mathbb{R}\right)$, we need to determine the properties of their growth functions.

Lemma 5.4. If $\mathrm{Z}: \operatorname{Conv}\left(\mathbb{R}^{n}, \mathbb{R}\right) \rightarrow[0,+\infty)$ is a continuous, $\mathrm{SL}(n)$ and translation invariant valuation, then its growth function $\psi_{1}$ satisfies $\lim _{t \rightarrow+\infty} \psi_{1}(t)=0$ and there exists $T \in \mathbb{R}$ such that $\psi_{2}(t)=0$ for all $t \geq T$.

Proof. Fix $0<\delta<\frac{1}{n-2}$ if $n \geq 3$ and $0<\delta<1$ if $n=2$ as well as $0<\rho<1$. For $b>0$ and $t \geq b$ let $T_{\delta}, P_{\delta, \rho}^{b, t}$ and $x_{\delta}$ be as in Lemma 2.2 and define $u_{\delta, \rho}^{b} \in \operatorname{Conv}\left(\mathbb{R}^{n}, \mathbb{R}\right)$ as

$$
\text { epi } u_{\delta, \rho}^{b}=\text { epi } \ell_{T_{\delta}} \cap\left\{x_{1} \leq b(1+\delta)+\rho\left(x_{n+1}-b\right)\right\} \text {. }
$$

That is

$$
\left\{u_{\delta, \rho}^{b} \leq t\right\}= \begin{cases}\emptyset, & \text { if } t<0 \\ t T_{\delta}, & \text { if } 0 \leq t<b \\ P_{\delta, \rho}^{b, t}, & \text { if } t \geq b .\end{cases}
$$

Hence, by Lemma 2.2 we have

$$
u_{\delta, \rho}^{b} \wedge\left(\ell_{T_{\delta}} \circ \tau_{b x_{\delta}}^{-1}+b\right)=\ell_{T_{\delta}} \quad \text { and } \quad u_{\delta, \rho}^{b} \vee\left(\ell_{T_{\delta}} \circ \tau_{b x_{\delta}}^{-1}+b\right)=\ell_{T_{\delta} \cap\left\{x_{1} \leq \rho\right\}} \circ \tau_{b x_{\delta}}^{-1}+b,
$$

where $\tau_{b x_{\delta}}$ denotes the translation $x \mapsto x+b x_{\delta}$ and where all occurring functions are elements of $\operatorname{Conv}\left(\mathbb{R}^{n}, \mathbb{R}\right)$. Hence, translation invariance together with the valuation property of $\mathrm{Z}$ shows

$$
\mathrm{Z}\left(u_{\delta, \rho}^{b}\right)+\mathrm{Z}\left(\ell_{T_{\delta}}+b\right)=\mathrm{Z}\left(\ell_{T_{\delta}}\right)+\mathrm{Z}\left(\ell_{T_{\delta} \cap\left\{x_{1} \leq \rho\right\}}+b\right) .
$$


By Lemma 3.2 and (12) we have $u_{\delta, \rho}^{b} \stackrel{e p i}{\longrightarrow} \ell_{T_{\delta}}$ as $b \rightarrow+\infty$. By the continuity of Z and Lemma 5.2 we now have

$$
\begin{aligned}
0 & =\lim _{b \rightarrow+\infty} \mathrm{Z}\left(\ell_{T_{\delta}}\right)-\mathrm{Z}\left(u_{\delta, \rho}^{b}\right) \\
& =\lim _{b \rightarrow+\infty}\left(\mathrm{Z}\left(\ell_{T_{\delta}}+b\right)-\mathrm{Z}\left(\ell_{T_{\delta} \cap\left\{x_{1} \leq \rho\right\}}+b\right)\right) \\
& =\lim _{b \rightarrow+\infty}\left(\psi_{1}(b)\left(V_{n}\left(T_{\delta}\right)-V_{n}\left(T_{\delta} \cap\left\{x_{1} \leq \rho\right\}\right)\right)+\psi_{2}(b)\left(V_{n}^{*}\left(T_{\delta}\right)-V_{n}^{*}\left(T_{\delta} \cap\left\{x_{1} \leq \rho\right\}\right)\right)\right) .
\end{aligned}
$$

Repeating the construction above but composing each of the occurring functions with $x \mapsto \frac{x}{2}$ and considering that $V_{n}$ and $V_{n}^{*}$ have different degrees of homogeneity, one easily deduces that $\lim _{b \rightarrow+\infty} \psi_{1}(b)=\lim _{b \rightarrow+\infty} \psi_{2}(b)=0$.

Assume now that there does not exist $T \in \mathbb{R}$ as claimed. In this case, there exists a sequence $t_{k}, k \in \mathbb{N}$ such that $t_{k}<t_{k+1}, \lim _{k \rightarrow+\infty} t_{k}=+\infty$ and $\psi_{2}\left(t_{k}\right)>0$ for every $k \in \mathbb{N}$. We repeat the construction above with $b=t_{k}$ and $\rho=\rho_{k}:=\left(1+\psi_{2}\left(t_{k}\right)^{-1}\right)^{-1}$ for $k \in \mathbb{N}$. Again, we have $u_{\delta, \rho_{k}}^{t_{k}} \stackrel{e p i}{\longrightarrow} \ell_{T_{\delta}}$ as $k \rightarrow+\infty$. Using the continuity of $\mathrm{Z}$ and the second part of Lemma 2.2 this gives

$$
\begin{aligned}
0 & =\lim _{k \rightarrow+\infty} \mathrm{Z}\left(\ell_{T_{\delta}}+t_{k}\right)-\mathrm{Z}\left(\ell_{T_{\delta} \cap\left\{x_{1} \leq \rho_{k}\right\}}+t_{k}\right) \\
& =\lim _{k \rightarrow+\infty} \psi_{1}\left(t_{k}\right)\left(V_{n}\left(T_{\delta}\right)-V_{n}\left(T_{\delta} \cap\left\{x_{1} \leq \rho_{k}\right\}\right)\right)+\psi_{2}\left(t_{k}\right)\left(V_{n}^{*}\left(T_{\delta}\right)-V_{n}^{*}\left(T_{\delta} \cap\left\{x_{1} \leq \rho_{k}\right\}\right)\right) \\
& =0-\lim _{k \rightarrow+\infty} \psi_{2}\left(t_{k}\right)\left(\frac{1}{n ! \delta^{n-2}} \frac{1+\delta}{\delta(1-(n-2) \delta)}\left(1+\frac{1}{\psi_{2}\left(t_{k}\right)}-\frac{1}{1+\delta}\right)\right) \\
& =\frac{1}{n ! \delta^{n-2}} \frac{1+\delta}{\delta(1-(n-2) \delta)},
\end{aligned}
$$

which is a contradiction.

Lemma 5.5. Let $\mathrm{Y}$ be a continuous and translation invariant valuation on $\operatorname{Conv}\left(\mathbb{R}^{n}, \mathbb{R}\right)$. For every $\delta>0$ the map

$$
u \mapsto \mathrm{Y}_{\delta}(u):=\mathrm{Y}\left(\operatorname{reg}_{\delta} u\right)
$$

defines a continuous and translation invariant valuation on $\operatorname{Conv}\left(\mathbb{R}^{n}\right)$. Furthermore, for $0 \leq$ $i \leq n$, there exist $\varphi_{i, \delta}: \mathbb{R} \times \mathcal{K}^{n} \rightarrow \mathbb{R}$ such that for every $t \in \mathbb{R}$, the map $K \mapsto \varphi_{i, \delta}(t, K)$ is a continuous, translation invariant, $i$-simple valuation that is homogeneous of degree $i$ and

$$
\mathrm{Y}_{\delta}\left(\mathrm{I}_{K}+t\right)=\sum_{i=0}^{n} \varphi_{i, \delta}(t, K)
$$

for every $K \in \mathcal{K}^{n}$ and $t \in \mathbb{R}$. Moreover, the maps $\delta \mapsto \varphi_{i, \delta}(t, K)$ and $t \mapsto \varphi_{i, \delta}(t, K)$ are continuous and if $\mathrm{Y}$ is non-negative, then so are $\mathrm{Y}_{\delta}$ and $\varphi_{i, \delta}$.

Proof. For $u, v \in \operatorname{Conv}\left(\mathbb{R}^{n}\right)$ such that $u \wedge v \in \operatorname{Conv}\left(\mathbb{R}^{n}\right)$, we have by Lemma 3.12 and the valuation property of $\mathrm{Y}$

$$
\begin{aligned}
\mathrm{Y}_{\delta}(u \wedge v)+\mathrm{Y}_{\delta}(u \vee v) & =\mathrm{Y}\left(\operatorname{reg}_{\delta}(u \wedge v)\right)+\mathrm{Y}\left(\operatorname{reg}_{\delta}(u \vee v)\right) \\
& =\mathrm{Y}\left(\operatorname{reg}_{\delta} u \wedge \operatorname{reg}_{\delta} v\right)+\mathrm{Y}\left(\operatorname{reg}_{\delta} u \vee \operatorname{reg}_{\delta} v\right) \\
& =\mathrm{Y}\left(\operatorname{reg}_{\delta} u\right)+\mathrm{Y}\left(\operatorname{reg}_{\delta} v\right) \\
& =\mathrm{Y}_{\delta}(u)+\mathrm{Y}_{\delta}(v),
\end{aligned}
$$


which shows the valuation property of $\mathrm{Y}_{\delta}$. Similar, one shows that $\mathrm{Y}_{\delta}$ is continuous and translation invariant.

For $t \in \mathbb{R}$ and $\delta>0$, define $\mu_{\delta, t}: \mathcal{K}^{n} \rightarrow \mathbb{R}$ as

$$
\mu_{\delta, t}(K)=\mathrm{Y}_{\delta}\left(\mathrm{I}_{K}+t\right)
$$

By the properties of $\mathrm{Y}_{\delta}$ it is easy to see that $\mu_{\delta, t}$ defines a continuous and translation invariant valuation on $\mathcal{K}^{n}$. By Theorem 2.1 there exist continuous, translation invariant, $i$-simple valuations $\left(\mu_{\delta, t}\right)_{i}$ that are homogeneous of degree $i, 0 \leq i \leq n$, such that $\mu_{\delta, t}=\left(\mu_{\delta, t}\right)_{0}+\cdots+\left(\mu_{\delta, t}\right)_{n}$. Since $t \in \mathbb{R}$ and $\delta>0$ were arbitrary, this defines functions $\varphi_{i, \delta}(t, K)=\left(\mu_{\delta, t}\right)_{i}(K)$ for $0 \leq i \leq n$. As $t \mapsto \mathrm{Y}_{\delta}\left(\mathrm{I}_{K}+t\right)$ is continuous, it is easy to see that the maps $t \mapsto \varphi_{i, \delta}(t, K)$ are continuous as well. Furthermore, by (7) and Lemma 3.2 the map $\delta \mapsto \mathrm{Y}_{\delta}\left(\mathrm{I}_{K}+t\right)$ is continuous, which together with homogeneity shows that also the maps $\delta \mapsto \varphi_{i, \delta}(t, K)$ are continuous.

Finally, if $\mathrm{Y}$ is non-negative, then by definition also $\mathrm{Y}_{\delta}$ is non-negative and consequently the valuations $\varphi_{i, \delta}$ are non-negative as well, which can be seen by evaluating at convex bodies $K$ of different dimensions.

Lemma 5.6. Let $\mathrm{Z}$ be a non-negative, continuous, $\mathrm{SL}(n)$ and translation invariant valuation on $\operatorname{Conv}\left(\mathbb{R}^{n}, \mathbb{R}\right)$ and let $\psi_{0}, \psi_{1}$ and $\psi_{2}$ denote its growth functions. The map

$$
\mathrm{Y}(u)=\mathrm{Z}(u)-\psi_{0}\left(\min _{x \in \mathbb{R}^{n}} u(x)\right)-\int_{\operatorname{dom} u^{*}} \psi_{2}\left(\nabla u^{*}(x) \cdot x-u^{*}(x)\right) \mathrm{d} x
$$

defines a continuous, $\mathrm{SL}(n)$ and translation invariant valuation on $\operatorname{Conv}\left(\mathbb{R}^{n}, \mathbb{R}\right)$. Furthermore, if $\varphi_{i, \delta}: \mathbb{R} \times \mathcal{K}^{n} \rightarrow \mathbb{R}$ are given as in Lemma 5.5 such that

$$
\mathrm{Y}_{\delta}\left(\mathrm{I}_{K}+t\right)=\sum_{i=0}^{n} \varphi_{i, \delta}(t, K),
$$

then $\varphi_{i, \delta}$ is non-negative for every $1 \leq i \leq n$ and $\delta>0$. Moreover,

$$
\mathrm{Y}_{\delta}\left(\ell_{K}+t\right)=\psi_{1}(t) V_{n}\left(K_{\delta}\right)
$$

for every $K \in \mathcal{K}_{o}^{n}$ and $t \in \mathbb{R}$, where $K_{\delta}=\operatorname{conv}\left(K \cup \delta C^{n}\right)$.

Proof. By Lemma 4.1 and Lemma 4.6 the operator Y defines a continuous, SL $(n)$ and translation invariant valuation on $\operatorname{Conv}\left(\mathbb{R}^{n}, \mathbb{R}\right)$. By Lemma 5.2 we have

$$
\begin{aligned}
\mathrm{Y}\left(\ell_{L}+t\right) & =\mathrm{Z}\left(\ell_{L}+t\right)-\psi_{0}(t)-\int_{L^{*}} \psi_{2}(t) \mathrm{d} x \\
& =\psi_{0}(t)+\psi_{1}(t) V_{n}(L)+\psi_{2}(t) V_{n}^{*}(L)-\psi_{0}(t)-\psi_{2}(t) V_{n}^{*}(L) \\
& =\psi_{1}(t) V_{n}(L)
\end{aligned}
$$

for every $L \in \mathcal{K}_{(o)}^{n}$. Therefore, by Lemma 3.12

$$
\mathrm{Y}_{\delta}\left(\ell_{K}+t\right)=\mathrm{Y}\left(\operatorname{reg}_{\delta} \ell_{K}+t\right)=\mathrm{Y}\left(K_{\delta}+t\right)=\psi_{1}(t) V_{n}\left(K_{\delta}\right)
$$


for every $K \in \mathcal{K}_{o}^{n}$.

For arbitrary $t \in \mathbb{R}$ and $\delta>0$ observe that

$$
K \mapsto \psi_{0}\left(\min _{x \in \mathbb{R}^{n}} \operatorname{reg}_{\delta} \mathrm{I}_{K}(x)+t\right)=\psi_{0}(t)
$$

is a translation invariant valuation on $\mathcal{K}^{n}$ that is homogeneous of degree 0 . Furthermore, for any $K \in \mathcal{K}^{n}$ one has

$$
\nabla h(K, x) \cdot x=h(K, x)
$$

for a.e. $x \in \mathbb{R}^{n}$. Setting $u_{K}=\operatorname{reg}_{\delta} \mathrm{I}_{K}+t$ with $K \in \mathcal{K}^{n}$ we have

$$
u_{K}^{*}=\left(\mathrm{I}_{K}+t\right)^{*}+\mathrm{I}_{\delta^{-1} Q^{n}}=h(K, \cdot)-t+\mathrm{I}_{\delta^{-1} Q^{n}}
$$

which shows that also

$$
\begin{aligned}
K & \mapsto \int_{\operatorname{dom} u_{K}^{*}} \psi_{2}\left(\nabla u_{K}^{*}(x) \cdot x-u_{K}^{*}(x)\right) \mathrm{d} x \\
& =\int_{\delta^{-1} Q^{n}} \psi_{2}(\nabla h(K, x) \cdot x-h(K, x)+t) \mathrm{d} x \\
& =\delta^{n} V_{n}\left(Q^{n}\right) \psi_{2}(t),
\end{aligned}
$$

is a translation invariant valuation on $\mathcal{K}^{n}$ that is homogeneous of degree 0 . Hence, if we apply Lemma 5.5 to both $\mathrm{Y}$ and $\mathrm{Z}$ we obtain

$$
\begin{aligned}
\sum_{i=0}^{n} \varphi_{i, \delta}(t, K) & =\mathrm{Y}_{\delta}\left(\mathrm{I}_{K}+t\right) \\
& =\mathrm{Z}_{\delta}\left(\mathrm{I}_{K}+t\right)-\psi_{0}(t)-\delta^{n} V_{n}\left(Q^{n}\right) \psi_{2}(t) \\
& =\sum_{i=0}^{n} \rho_{i, \delta}(t, K)-\psi_{0}(t)-\delta^{n} V_{n}\left(Q^{n}\right) \psi_{2}(t)
\end{aligned}
$$

with $\rho_{i, \delta}: \mathbb{R} \times \mathcal{K}^{n} \rightarrow[0,+\infty)$. By homogeneity, we must have $\varphi_{i, \delta}=\rho_{i, \delta}$ for every $1 \leq i \leq n$ and $\delta>0$ and in particular, those maps are non-negative.

By Lemma 5.3 every non-negative, continuous, $\mathrm{SL}(n)$ and translation invariant valuation $\mathrm{Z}$ on $\operatorname{Conv}\left(\mathbb{R}^{n}, \mathbb{R}\right)$ is uniquely determined by its growth functions $\psi_{0}, \psi_{1}, \psi_{2}: \mathbb{R} \rightarrow[0,+\infty)$ and by Lemma 5.4 we know that $\lim _{t \rightarrow+\infty} \psi_{1}(t)=0$. In Lemma 5.7 and Lemma 5.8 we will furthermore show that $\psi_{1}$ is $n$-times continuously differentiable and that its $n$-th derivative has constant sign. The idea of the proof of Lemma 5.7 is to describe the behaviour of $\mathrm{Z}$ on regularizations of indicator functions with the help of $\psi_{1}$. As the proof is rather technical, we will describe here its basic idea in the 1-dimensional case.

Let $\mathrm{Y}_{\delta}$ be defined as in Lemma 5.6 and recall that $\mathrm{Y}_{\delta}\left(\ell_{K}+t\right)=\psi_{1}(t) V_{n}\left(K_{\delta}\right)$ for every $K \in \mathcal{K}_{o}^{1}$ and $t \in \mathbb{R}$. We will now compute $\mathrm{Y}_{\delta}\left(\mathrm{I}_{[0, \lambda]}+t\right)$ for $\lambda>0$ and $t \in \mathbb{R}$. By the properties of $\mathrm{Y}_{\delta}$ we have

$$
\begin{aligned}
\mathrm{Y}_{\delta}\left(\ell_{[0, \lambda / h]}+t\right) & =\psi_{1}(t) V_{1}\left(\left[0, \frac{\lambda}{h}\right]_{\delta}\right) \\
& =\psi_{1}(t) V_{1}\left(\operatorname{conv}\left(\left[0, \frac{\lambda}{h}\right] \cup[-\delta, \delta]\right)\right) \\
& =\psi_{1}(t)\left(\max \left\{\frac{\lambda}{h}, \delta\right\}+\delta\right)
\end{aligned}
$$


for every $\lambda, h>0$ and $t \in \mathbb{R}$. Let $v^{\lambda, h}=\ell_{[0, \lambda / h]}+\mathrm{I}_{[0, \lambda]}$, that is $v^{\lambda, h}(x)=h x / \lambda$ for $0 \leq x \leq \lambda$ and $v^{\lambda, h}(x)=+\infty$ if $x<0$ or $x>\lambda$. Note that $v^{\lambda, h} \stackrel{e p i}{\longrightarrow} \mathrm{I}_{[0, \lambda]}$ as $h \rightarrow 0^{+}$. In order to calculate $\mathrm{Y}_{\delta}\left(v^{\lambda, h}+t\right)$, let $\tau_{\lambda}$ denote the translation $x \mapsto x+\lambda$ and obverse that

$$
v^{\lambda, h} \wedge\left(\ell_{[0, \lambda / h]} \circ \tau_{\lambda}^{-1}+h\right)=\ell_{[0, \lambda / h]}
$$

and

$$
v^{\lambda, h} \vee\left(\ell_{[0, \lambda / h]} \circ \tau_{\lambda}^{-1}+h\right)=\mathrm{I}_{\{\lambda\}}+h .
$$

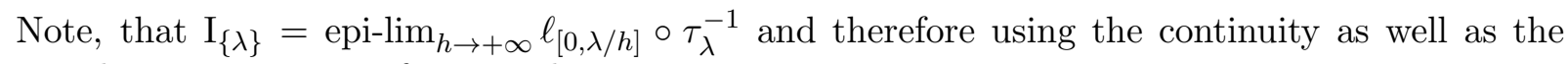
translation invariance of $\mathrm{Y}_{\delta}$ we obtain

$$
\begin{aligned}
\mathrm{Y}_{\delta}\left(\mathrm{I}_{\{\lambda\}}+t\right) & =\lim _{h \rightarrow+\infty} \mathrm{Y}_{\delta}\left(\ell_{[0, \lambda / h]} \circ \tau_{\lambda}^{-1}+t\right) \\
& =\lim _{h \rightarrow+\infty} \psi_{1}(t)\left(\max \left\{\frac{\lambda}{h}, \delta\right\}+\delta\right) \\
& =2 \delta \psi_{1}(t)
\end{aligned}
$$

for every $\delta, \lambda>0$ and $t \in \mathbb{R}$. Thus, we have by the valuation property of $\mathrm{Y}_{\delta}$ together with translation invariance that

$$
\begin{aligned}
\mathrm{Y}_{\delta}\left(v^{\lambda, h}+t\right) & =\mathrm{Y}_{\delta}\left(\ell_{[0, \lambda / h]}+t\right)+\mathrm{Y}_{\delta}\left(\mathrm{I}_{\lambda}+h+t\right)-\mathrm{Y}_{\delta}\left(\ell_{[0, \lambda / h]} \circ \tau_{\lambda}^{-1}+h+t\right) \\
& =\left(\psi_{1}(t)-\psi_{1}(t+h)\right)\left(\max \left\{\frac{\lambda}{h}, \delta\right\}+\delta\right)+2 \delta \psi_{1}(t+h)
\end{aligned}
$$

By continuity, this gives

$$
\begin{aligned}
\mathrm{Y}_{\delta}\left(\mathrm{I}_{[0, \lambda]}+t\right) & =\lim _{h \rightarrow 0^{+}} \mathrm{Y}_{\delta}\left(v^{\lambda, h}+t\right) \\
& =\lim _{h \rightarrow 0^{+}}\left(\lambda \frac{\psi_{1}(t)-\psi_{1}(t+h)}{h}+\delta\left(\psi_{1}(t)-\psi_{1}(t+h)\right)+2 \delta \psi_{1}(t+h)\right) \\
& =\lambda \lim _{h \rightarrow 0^{+}} \frac{\psi_{1}(t)-\psi_{1}(t+h)}{h}+2 \delta \psi_{1}(t),
\end{aligned}
$$

which shows that $\psi_{1}$ is differentiable from the right. Similarly, one has

$$
\begin{aligned}
\mathrm{Y}_{\delta}\left(\mathrm{I}_{[0, \lambda]}+t\right) & =\lim _{h \rightarrow 0^{+}} \mathrm{Y}_{\delta}\left(v^{\lambda, h}+t-h\right) \\
& =\lambda \lim _{h \rightarrow 0^{+}} \frac{\psi_{1}(t-h)-\psi_{1}(t)}{h}+2 \delta \psi_{1}(t),
\end{aligned}
$$

which shows that $\psi_{1}$ is differentiable from the left and thus

$$
\mathrm{Y}_{\delta}\left(\mathrm{I}_{[0, \lambda]}+t\right)=-\lambda \psi_{1}^{\prime}(t)+2 \delta \psi_{1}(t)
$$

for every $\delta, \lambda>0$ and $t \in \mathbb{R}$.

Lemma 5.7. Let $\mathrm{Y}_{\delta}$ be defined as in Lemma 5.6. For $\lambda>0$,

$$
\mathrm{Y}_{\delta}\left(\mathrm{I}_{[0, \lambda]^{n}}+t\right)=\frac{1}{n !} \sum_{k=0}^{n} c_{n, k}(\delta)(-\lambda)^{k} \psi_{1}^{(k)}(t),
$$

for every $t \in \mathbb{R}$, where $c_{n, k}(\delta)$ are polynomials in $\delta$ with $c_{n, n}(\delta) \equiv 1$ and $\psi_{1}^{(i)}(t)=\frac{\mathrm{d}^{i}}{\mathrm{~d} t^{i}} \psi_{1}(t)$ for $i \geq 0$. In particular, $\psi_{1}$ is n-times continuously differentiable. 
Proof. Let $\left\{e_{1}, \ldots, e_{n}\right\}$ denote the standard basis of $\mathbb{R}^{n}$ and set $e_{0}=0$. For $h=\left(h_{1}, \ldots, h_{n}\right)$, $\lambda>0$ and $0 \leq i<n$, define the function $u_{i}^{\lambda, h}$ through its sublevel sets as

$$
\left\{u_{i}^{\lambda, h}<0\right\}=\emptyset, \quad\left\{u_{i}^{\lambda, h} \leq s\right\}=\left[0, \lambda e_{0}\right]+\cdots\left[0, \lambda e_{i}\right]+\operatorname{conv}\left\{0, s \lambda e_{i+1} / h_{i+1}, \ldots, s \lambda e_{n} / h_{n}\right\},
$$

for every $s \geq 0$. Note, that $u_{i}^{\lambda, h}$ does not depend on $h_{j}$ for $j \leq i$. Furthermore, let $u_{n}^{\lambda, h}=\mathrm{I}_{[0, \lambda]^{n}}$. We will use induction to show that $u_{i}^{\lambda, h} \in \operatorname{Conv}\left(\mathbb{R}^{n}\right)$ and

$$
\mathrm{Y}_{\delta}\left(u_{i}^{\lambda, h}+t\right)=\frac{1}{n !}\left(\sum_{k=0}^{i} c_{i, k}(\delta)(-\lambda)^{k} \psi_{1}^{(k)}(t)\right) \prod_{l=i+1}^{n}\left(\max \left\{\frac{\lambda}{h_{l}}, \delta\right\}+\delta\right),
$$

for every $t \in \mathbb{R}, \lambda>0$ and $0 \leq i \leq n$ with polynomials $c_{i, k}$ such that $c_{i, i}(\delta) \equiv 1$.

For $i=0$, let $P_{h}=\operatorname{conv}\left\{0, e_{1} / h_{1}, \ldots, e_{n} / h_{n}\right\} \in \mathcal{P}_{o}^{n}$. Observe, that $u_{0}^{\lambda, h}=\ell_{\lambda P_{h}} \in \operatorname{Conv}\left(\mathbb{R}^{n}\right)$. Hence, by the properties of $\mathrm{Y}_{\delta}$ and Lemma 2.3 we have

$$
\begin{aligned}
\mathrm{Y}_{\delta}\left(u_{0}^{\lambda, h}+t\right)=\mathrm{Y}_{\delta}\left(\ell_{\lambda P_{h}}+t\right) & =\psi_{1}(t) V_{n}\left(\left(\lambda P_{h}\right)_{\delta}\right) \\
& =\psi_{1}(t) V_{n}\left(\operatorname{conv}\left(\delta C^{n} \cup \lambda P_{h}\right)\right) \\
& =\psi_{1}(t) \frac{1}{n !} \prod_{k=1}^{n}\left(\max \left\{\frac{\lambda}{h_{k}}, \delta\right\}+\delta\right),
\end{aligned}
$$

for every $t \in \mathbb{R}$.

Now assume that the statement holds true for $i \geq 0$. Define the function $v_{i+1}^{\lambda, h}$ by

$$
\left\{v_{i+1}^{\lambda, h} \leq s\right\}=\left\{u_{i}^{\lambda, h} \leq s\right\} \cap\left\{x_{i+1} \leq \lambda\right\},
$$

for every $s \in \mathbb{R}$. As epi $v_{i+1}^{\lambda, h}=\operatorname{epi} u_{i}^{\lambda, h} \cap\left\{x_{i+1} \leq \lambda\right\}$, it is easy to see that $v_{i+1}^{\lambda, h} \in \operatorname{Conv}\left(\mathbb{R}^{n}\right)$. Furthermore, by convergence of level sets and Lemma 3.2 we have $v_{i+1}^{\lambda, h} \stackrel{e p i}{\longrightarrow} u_{i+1}^{\lambda, h}$ as $h_{i+1} \rightarrow 0^{+}$. Since epi-limits of convex functions are again convex (Theorem 3.3 ), it is easy to see that $u_{i+1}^{h} \in$ $\operatorname{Conv}\left(\mathbb{R}^{n}\right)$. Now, let $\tau_{i+1}^{\lambda}$ be the translation $x \mapsto x+\lambda e_{i+1}$. Note, that

$$
\left\{v_{i+1}^{\lambda, h} \leq s\right\} \cup\left\{\left(u_{i}^{\lambda, h} \circ\left(\tau_{i+1}^{\lambda}\right)^{-1}+h_{i+1}\right) \leq s\right\}=\left\{u_{i}^{\lambda, h} \leq s\right\} .
$$

Furthermore, let

$$
w_{i+1}^{\lambda, h}:=v_{i+1}^{\lambda, h} \vee\left(u_{i}^{\lambda, h} \circ\left(\tau_{i+1}^{\lambda}\right)^{-1}+h_{i+1}\right) \in \operatorname{Conv}\left(\mathbb{R}^{n}\right)
$$

and observe that dom $w_{i+1}^{\lambda, h} \subset\left\{x_{i+1}=\lambda\right\}$ as well as

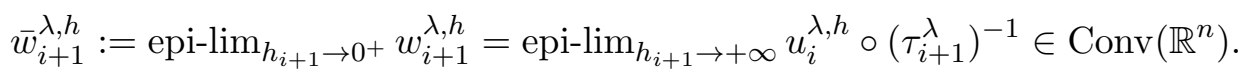

Hence, using the induction assumption and the continuity as well as the translation invariance of $\mathrm{Y}_{\delta}$, we obtain

$$
\begin{aligned}
\mathrm{Y}_{\delta}\left(\bar{w}_{i+1}^{\lambda, h}+t\right) & =\lim _{h_{i+1} \rightarrow+\infty} \mathrm{Y}_{\delta}\left(u_{i}^{\lambda, h}+t\right) \\
& =\lim _{h_{i+1} \rightarrow+\infty} \frac{1}{n !}\left(\sum_{k=0}^{i} c_{i, k}(\delta)(-\lambda)^{k} \psi_{1}^{(k)}(t)\right) \prod_{l=i+1}^{n}\left(\max \left\{\frac{\lambda}{h_{l}}, \delta\right\}+\delta\right) \\
& =\frac{2 \delta}{n !}\left(\sum_{k=0}^{i} c_{i, k}(\delta)(-\lambda)^{k} \psi_{1}^{(k)}(t)\right) \prod_{l=i+2}^{n}\left(\max \left\{\frac{\lambda}{h_{l}}, \delta\right\}+\delta\right) .
\end{aligned}
$$


Furthermore, (13) and (14) together with the valuation property of $\mathrm{Y}_{\delta}$ give

$$
\mathrm{Y}_{\delta}\left(u_{i}^{\lambda, h}+t\right)+\mathrm{Y}_{\delta}\left(w_{i+1}^{\lambda, h}+t\right)=\mathrm{Y}_{\delta}\left(v_{i+1}^{\lambda, h}+t\right)+\mathrm{Y}_{\delta}\left(u_{i}^{\lambda, h} \circ\left(\tau_{i+1}^{\lambda}\right)^{-1}+h_{i+1}+t\right) .
$$

Using the induction assumption and the translation invariance of $\mathrm{Y}_{\delta}$ again, we obtain

$$
\begin{aligned}
\mathrm{Y}_{\delta}\left(v_{i+1}^{\lambda, h}+t\right)= & \mathrm{Y}_{\delta}\left(u_{i}^{\lambda, h}+t\right)-\mathrm{Y}_{\delta}\left(u_{i}^{\lambda, h}+t+h_{i+1}\right)+\mathrm{Y}_{\delta}\left(w_{i+1}^{\lambda, h}+t\right) \\
= & \frac{1}{n !}\left(\sum_{k=0}^{i} c_{i, k}(\delta)(-\lambda)^{k}\left(\psi_{1}^{(k)}(t)-\psi_{1}^{(k)}\left(t+h_{i+1}\right)\right)\right) \prod_{l=i+1}^{n}\left(\max \left\{\frac{\lambda}{h_{l}}, \delta\right\}+\delta\right) \\
& +\mathrm{Y}_{\delta}\left(w_{i+1}^{\lambda, h}+t\right) .
\end{aligned}
$$

As $h_{i+1} \rightarrow 0^{+}$, the continuity of $\mathrm{Y}_{\delta}$ gives

$$
\begin{aligned}
\mathrm{Y}_{\delta}\left(u_{i+1}^{\lambda, h}+t\right)= & \lim _{h_{i+1} \rightarrow 0^{+}} \mathrm{Y}_{\delta}\left(v_{i+1}^{\lambda, h}+t\right) \\
= & \frac{1}{n !}\left(\sum_{k=0}^{i} c_{i, k}(\delta)(-\lambda)^{k} \lambda \lim _{h_{i+1} \rightarrow 0^{+}} \frac{\psi_{1}^{(k)}(t)-\psi_{1}^{(k)}\left(t+h_{i+1}\right)}{h_{i+1}}\right) \prod_{l=i+2}^{n}\left(\max \left\{\frac{\lambda}{h_{l}}, \delta\right\}+\delta\right) \\
& +\mathrm{Y}_{\delta}\left(\bar{w}_{i+1}^{\lambda, h}+t\right),
\end{aligned}
$$

which shows that $\psi_{1}^{(i)}$ is differentiable from the right. Similarly, using $v_{i+1}^{\lambda, h}+t-h_{i+1} \stackrel{e p i}{\longrightarrow} u_{i+1}^{\lambda, h}+t$ as $h_{i+1} \rightarrow 0^{+}$, shows that $\psi_{1}^{(i)}$ is differentiable from the left. Collecting terms therefore shows for every $t \in \mathbb{R}$

$$
\begin{aligned}
\mathrm{Y}_{\delta}\left(u_{i+1}^{\lambda, h}+t\right) & =\frac{1}{n !}\left(\sum_{k=0}^{i} c_{i, k}(\delta)\left((-\lambda)^{k+1} \psi_{1}^{(k+1)}(t)+2 \delta(-\lambda)^{k} \psi_{1}^{(k)}(t)\right)\right) \prod_{l=i+2}^{n}\left(\max \left\{\frac{\lambda}{h_{l}}, \delta\right\}+\delta\right) \\
& \left.=\frac{1}{n !}\left(\sum_{k=0}^{i+1} c_{i+1, k}(\delta)(-\lambda)^{k} \psi_{1}^{(k)}(t)\right)\right) \prod_{l=i+2}^{n}\left(\max \left\{\frac{\lambda}{h_{l}}, \delta\right\}+\delta\right)
\end{aligned}
$$

with $c_{i+1,1}(\delta)=2 \delta, c_{i+1, k}(\delta)=c_{i, k-1}(\delta)+2 \delta c_{i, k}(\delta)$ for $1<k<i+1$ and $c_{i+1, i+1}(\delta)=c_{i, i}(\delta) \equiv$ 1 .

Lemma 5.8. Let $\mathrm{Z}$ be a non-negative, continuous, $\mathrm{SL}(n)$ and translation invariant valuation on $\operatorname{Conv}\left(\mathbb{R}^{n}, \mathbb{R}\right)$. If $\psi_{1}$ denotes the growth function of $\mathrm{Z}$, then $\psi_{1}$ is n-times continuously differentiable and $(-1)^{n} \psi_{1}^{(n)}$ is non-negative.

Proof. By Lemma 5.6, we have for $\lambda, \delta>0$ and $t \in \mathbb{R}$.

$$
\mathrm{Y}_{\delta}\left(\mathrm{I}_{[0, \lambda]^{n}}+t\right)=\sum_{k=0}^{n} \lambda^{k} \varphi_{k, \delta}\left(t,[0,1]^{n}\right),
$$

where $\varphi_{k, \delta}\left(t,[0,1]^{n}\right)$ is non-negative for every $1 \leq k \leq n$. Furthermore, by Lemma 5.7, we have

$$
\mathrm{Y}_{\delta}\left(\mathrm{I}_{[0, \lambda]^{n}}+t\right)=\frac{1}{n !} \sum_{k=0}^{n} \lambda^{k}(-1)^{k} c_{n, k}(\delta) \psi_{1}^{(k)}(t),
$$

with $c_{n, n}(\delta) \equiv 1$. The result now follows by equating coefficients. 
Lemma 5.9. Let $\zeta: \mathbb{R} \rightarrow \mathbb{R}$ be $n$-times continuously differentiable. If $\lim _{t \rightarrow+\infty} \zeta(t)=0$ and $\zeta^{(n)}$ has constant sign on $\left[t_{0},+\infty\right)$ for some $t_{0} \in \mathbb{R}$, then

$$
\int_{0}^{+\infty} r^{n-1} \frac{(-1)^{n}}{(n-1) !} \zeta^{(n)}(r+t) \mathrm{d} r=\zeta(t)
$$

for every $t \in \mathbb{R}$. In particular, $\zeta^{(n)}$ has finite moment of order $n-1$. Moreover,

$$
\lim _{r \rightarrow+\infty} r^{k} \zeta^{(k)}(r+t)=0
$$

for every $0 \leq k \leq n-1$ and $t \in \mathbb{R}$.

Proof. Throughout the proof we will assume that $(-1)^{n} \zeta^{(n)}$ is non-negative on $\left[t_{0},+\infty\right)$, since we can always consider $-\zeta$ instead of $\zeta$. We use induction on $n$ and start with the case $n=1$. Integration by parts together with the assumption on $\zeta$ gives

$$
\begin{aligned}
\int_{0}^{+\infty}(-1) \zeta^{\prime}(r+t) \mathrm{d} r & =\lim _{R \rightarrow+\infty} \int_{0}^{R}(-1) \zeta^{\prime}(r+t) \mathrm{d} r \\
& =\lim _{R \rightarrow+\infty}(\zeta(t)-\zeta(t+R)) \\
& =\zeta(t),
\end{aligned}
$$

for every $t \in \mathbb{R}$.

Next, let $n \geq 2$ and assume that the statement holds true for $n-1$. Since $(-1)^{n} \zeta^{(n)}$ is non-negative on $\left[t_{0},+\infty\right)$, the function $(-1)^{n-1} \zeta^{(n-1)}$ is non-increasing on $\left[t_{0},+\infty\right)$ and therefore $(-1)^{n-1} \zeta^{(n-1)}$ has constant sign on $\left[t_{1},+\infty\right)$ for some $t_{1} \in \mathbb{R}$. Hence, by the induction hypothesis

$$
\int_{0}^{+\infty} r^{n-2} \frac{(-1)^{n-1}}{(n-2) !} \zeta^{(n-1)}(r+t) \mathrm{d} r=\zeta(t)
$$

and $\lim _{r \rightarrow+\infty} r^{k} \zeta^{(k)}(r+t)=0$ for every $0 \leq k \leq n-2$ and $t \in \mathbb{R}$. In particular, $\zeta^{(n-1)}$ has finite moment of order $n-2$ and therefore $\lim _{t \rightarrow+\infty} \zeta^{(n-1)}(t)=0$, which implies that $(-1)^{n-1} \zeta^{(n-1)}(t) \geq 0$ for every $t \geq t_{0}$. Using integration by parts, we obtain

$$
\begin{aligned}
\int_{0}^{R} r^{n-1} \frac{(-1)^{n}}{(n-1) !} \zeta^{(n)}(r & +t) \mathrm{d} r \\
& =R^{n-1} \frac{(-1)^{n}}{(n-1) !} \zeta^{(n-1)}(R+t)+\int_{0}^{R} r^{n-2} \frac{(-1)^{n-1}}{(n-2) !} \zeta^{(n-1)}(r+t) \mathrm{d} r
\end{aligned}
$$

for every $t \in \mathbb{R}$ and $R>0$. Since $r^{n-1} \frac{(-1)^{n}}{(n-1) !} \zeta^{(n)}(r+t) \geq 0$ for $r \geq \max \left\{0, t_{0}-t\right\}$, there exists

$$
C(t)=\lim _{R \rightarrow+\infty} \int_{0}^{R} r^{n-1} \frac{(-1)^{n}}{(n-1) !} \zeta^{(n)}(r+t) \mathrm{d} r \in(-\infty,+\infty],
$$

for every $t \in \mathbb{R}$. Hence, (15) implies that $R^{n-1} \frac{(-1)^{n}}{(n-1) !} \zeta^{(n-1)}(R+t)$ converges to

$$
\begin{aligned}
D(t) & =C(t)-\lim _{R \rightarrow+\infty} \int_{0}^{R} r^{n-2} \frac{(-1)^{n-1}}{(n-2) !} \zeta^{(n-1)}(r+t) \mathrm{d} r \\
& =C(t)-\zeta(t) \in(-\infty,+\infty]
\end{aligned}
$$


as $R \rightarrow+\infty$. Since $(-1)^{n-1} \zeta^{(n-1)}(R+t) \geq 0$ for every $R \geq t_{0}-t$ we have

$$
R^{n-1} \frac{(-1)^{n}}{(n-1) !} \zeta^{(n-1)}(R+t) \leq 0
$$

for every $R \geq \max \left\{0, t_{0}-t\right\}$ and therefore $D(t) \leq 0$, which is only possible if $C(t)<+\infty$ for every $t \in \mathbb{R}$.

It remains to show that $D(t) \equiv 0$. Assume on the contrary that there exists $\bar{t} \in \mathbb{R}$ such that $(n-1) ! D(\bar{t})=2 \eta<0$. It follows that

$$
r^{n-1}(-1)^{n} \zeta^{(n-1)}(r+\bar{t}) \leq \eta<0
$$

for every $r \geq r_{0}$ with $r_{0} \geq 0$ large enough and therefore

$$
(-1)^{n} \zeta^{(n-1)}(r+\bar{t}) \leq \frac{\eta}{r^{n-1}}
$$

Consequently,

$$
\begin{aligned}
(-1)^{n} \zeta^{(n-2)}(r+\bar{t}) & =(-1)^{n} \zeta^{(n-2)}\left(r_{0}+\bar{t}\right)+\int_{r_{0}}^{r}(-1)^{n} \zeta^{(n-1)}(s+\bar{t}) \mathrm{d} s \\
& \leq(-1)^{n} \zeta^{(n-2)}\left(r_{0}+\bar{t}\right)+\eta \int_{r_{0}}^{r} \frac{1}{s^{n-1}} \mathrm{~d} s \\
& =(-1)^{n} \zeta^{(n-2)}\left(r_{0}+\bar{t}\right)+\eta \begin{cases}\log (r)-\log \left(r_{0}\right), & n=2 \\
\frac{1}{n-2}\left(\frac{1}{r_{0}^{n-2}}-\frac{1}{r^{n-2}}\right), & n>2\end{cases}
\end{aligned}
$$

If $n=2$, this shows that

$$
\begin{aligned}
0 & =\lim _{r \rightarrow+\infty} \zeta(r+\bar{t}) \\
& \leq \zeta\left(r_{0}+\bar{t}\right)-\eta \log \left(r_{0}\right)+\eta \lim _{r \rightarrow+\infty} \log (r) \\
& =-\infty
\end{aligned}
$$

which is a contradiction. If $n>2$, we obtain

$$
\begin{aligned}
0 & =\lim _{r \rightarrow+\infty}(-1)^{n} r^{n-2} \zeta^{(n-2)}(r+\bar{t}) \\
& \leq-\frac{\eta}{n-2}+\lim _{r \rightarrow+\infty} r^{n-2}\left((-1)^{n} \zeta^{(n-2)}\left(r_{0}+\bar{t}\right)+\frac{\eta}{(n-2) r_{0}^{n-2}}\right) \\
& =-\frac{\eta}{n-2}+\lim _{r \rightarrow+\infty} r^{n-2} \frac{(-1)^{n}(n-2) r_{0}^{n-2} \zeta^{(n-2)}\left(r_{0}+\bar{t}\right)+\eta}{(n-2) r_{0}^{n-2}}
\end{aligned}
$$

which goes to $-\infty$ if $r_{0}$ is large enough. 


\subsection{Proof of the Theorem}

If $\zeta_{0}, \zeta_{1}, \zeta_{2}: \mathbb{R} \rightarrow[0,+\infty)$ are continuous functions such that $\zeta_{1}$ has finite moment of order $n-1$ and $\zeta_{2}(t)=0$ for all $t \geq T$ with some $T \in \mathbb{R}$, then Lemma 4.1, Lemma 4.2 and Lemma 4.6 show that

$$
u \mapsto \zeta_{0}\left(\min _{x \in \mathbb{R}^{n}} u(x)\right)+\int_{\mathbb{R}^{n}} \zeta_{1}(u(x)) \mathrm{d} x+\int_{\operatorname{dom} u^{*}} \zeta_{2}\left(\nabla u^{*}(x) \cdot x-u^{*}(x)\right) \mathrm{d} x,
$$

defines a non-negative, continuous, $\operatorname{SL}(n)$ and translation invariant valuation on $\operatorname{Conv}\left(\mathbb{R}^{n}, \mathbb{R}\right)$.

Conversely, let $\mathrm{Z}: \operatorname{Conv}\left(\mathbb{R}^{n}, \mathbb{R}\right) \rightarrow[0,+\infty)$ be a continuous, $\operatorname{SL}(n)$ and translation invariant valuation on $\operatorname{Conv}\left(\mathbb{R}^{n}, \mathbb{R}\right)$. By Lemma 5.2 the valuation $\mathrm{Z}$ has non-negative, continuous growth functions $\psi_{0}, \psi_{1}, \psi_{2}$. Lemma 5.4 shows that there exists $T \in \mathbb{R}$ such that $\psi_{2}(t)=0$ for all $t \geq T$. Furthermore, by Lemma 5.8 the function $\psi_{1}$ is $n$-times continuously differentiable and $\zeta_{1}(t):=\frac{(-1)^{n}}{n !} \psi_{1}^{(n)}(t)$ is non-negative. Moreover, Lemma 5.4 together with Lemma 5.9 shows that $\zeta_{1}$ has finite moment of order $n-1$ and

$$
n \int_{0}^{+\infty} r^{n-1} \zeta_{1}(r+t) \mathrm{d} r=\psi_{1}(t),
$$

for every $t \in \mathbb{R}$. Finally, for $u=\ell_{\lambda B^{n}}+t$ with $\lambda>0$ and $t \in \mathbb{R}$ we have $u^{*}=\mathrm{I}_{\lambda^{-1} B^{n}}-t$ and furthermore

$$
\begin{aligned}
\mathrm{Z}(u) & =\psi_{0}(t)+\psi_{1}(t) V_{n}\left(\lambda B^{n}\right)+\psi_{2}(t) V_{n}^{*}\left(\lambda B^{n}\right) \\
& =\psi_{0}(t)+\lambda^{n} V_{n}\left(B^{n}\right) \psi_{1}(t)+\psi_{2}(t) V_{n}\left(\lambda^{-1} B^{n}\right) \\
& =\psi_{0}(t)+\lambda^{n} n V_{n}\left(B^{n}\right) \int_{0}^{+\infty} r^{n-1} \zeta_{1}(r+t) \mathrm{d} r+\psi_{2}(t) V_{n}\left(\lambda^{-1} B^{n}\right) \\
& =\psi_{0}(t)+\lambda^{n} \int_{\mathbb{R}^{n}} \zeta_{1}(|x|+t) \mathrm{d} x+\psi_{2}(t) V_{n}\left(\lambda^{-1} B^{n}\right) \\
& =\psi_{0}(t)+\int_{\mathbb{R}^{n}} \zeta_{1}\left(\frac{|x|}{\lambda}+t\right) \mathrm{d} x+\int_{\lambda^{-1} B^{n}} \psi_{2}(t) \mathrm{d} x \\
& =\psi_{0}\left(\min _{x \in \mathbb{R}^{n}} u(x)\right)+\int_{\mathbb{R}^{n}} \zeta_{1}(u(x)) \mathrm{d} x+\int_{\operatorname{dom} u^{*}} \psi_{2}\left(\nabla u^{*}(x) \cdot x-u^{*}(x)\right) \mathrm{d} x .
\end{aligned}
$$

By the first part of the proof,

$$
u \mapsto \psi_{0}\left(\min _{x \in \mathbb{R}^{n}} u(x)\right)+\int_{\mathbb{R}^{n}} \zeta_{1}(u(x)) \mathrm{d} x+\int_{\operatorname{dom} u^{*}} \psi_{2}\left(\nabla u^{*}(x) \cdot x-u^{*}(x)\right) \mathrm{d} x
$$

defines a non-negative, continuous, $\operatorname{SL}(n)$ and translation invariant valuation on $\operatorname{Conv}\left(\mathbb{R}^{n}, \mathbb{R}\right)$ and by homogeneity it is easy to see that $\psi_{0}, \psi_{1}, \psi_{2}$ are its growth functions. Thus, Lemma 5.3 completes the proof of the Theorem.

\section{Acknowledgments}

The author would like to thank Jin Li for his helpful remarks regarding the proof of Lemma 5.1, 


\section{References}

[1] Abardia, J., And Wannerer, T. Aleksandrov-Fenchel inequalities for unitary valuations of degree 2 and 3. Calc. Var. Partial Differential Equations 54 (2015), 1767-1791.

[2] Alesker, S. Continuous rotation invariant valuations on convex sets. Ann. of Math. (2) 149 (1999), 9971005.

[3] Alesker, S. Description of translation invariant valuations on convex sets with solution of P. McMullen's conjecture. Geom. Funct. Anal. 11 (2001), 244-272.

[4] Alesker, S. Valuations on convex functions and convex sets and Monge-Ampère operators. Preprint (arXiv:1703.08778).

[5] Baryshnikov, Y., Ghrist, R., And Wright, M. Hadwiger's Theorem for definable functions. Adv. Math. 245 (2013), 573-586.

[6] Beer, G., Rockafellar, R.-T., and Wets, R. J.-B. A characterization of epi-convergence in terms of convergence of level sets. Proc. Amer. Math. Soc. 116 (1992), 753-761.

[7] Bernig, A., and Fu, J. H. G. Hermitian integral geometry. Ann. of Math. (2) 173 (2011), 907-945.

[8] Blaschke, W. Vorlesungen über Integralgeometrie. H. 2. Teubner, Berlin, 1937.

[9] Bobkov, S. G., Colesanti, A., And Fragalà, I. Quermassintegrals of quasi-concave functions and generalized Prékopa-Leindler inequalities. Manuscripta Math. 143 (2014), 131-169.

[10] Colesanti, A., And Fragalà, I. The first variation of the total mass of log-concave functions and related inequalities. Adv. Math. 244 (2013), 708-749.

[11] Colesanti, A., And Lombardi, N. Valuations on the space of quasi-concave functions. In Geometric Aspects of Functional Analysis: Israel Seminar (GAFA) 2014-2016, B. Klartag and E. Milman, Eds. Springer International Publishing, Cham, 2017, pp. 71-105.

[12] Colesanti, A., Lombardi, N., and Parapatits, L. Translation invariant valuations on quasi-concave functions. Studia Math. 243 (2018), 79-99.

[13] Colesanti, A., Ludwig, M., And Mussnig, F. Minkowski valuations on convex functions. Calc. Var. Partial Differential Equations 56 (2017), Art. 162.

[14] Colesanti, A., Ludwig, M., And Mussnig, F. Hessian valuations. Indiana Univ. Math. J. (in press).

[15] Colesanti, A., Ludwig, M., And Mussnig, F. Valuations on convex functions. Int. Math. Res. Not. IMRN (in press).

[16] Dal Maso, G. An Introduction to Г-Convergence, vol. 8 of Progress in Nonlinear Differential Equations and their Applications. Birkhäuser Boston, Inc., Boston, MA, 1993.

[17] Federer, H. Geometric Measure Theory. Classics in Mathematics. Springer-Verlag, Berlin Heidelberg, 1996.

[18] Gruber, P. M. Convex and Discrete Geometry, vol. 336 of Grundlehren der mathematischen Wissenschaften. Springer-Verlag, Berlin Heidelberg, 2007.

[19] Haberl, C. Minkowski valuations intertwining the special linear group. J. Eur. Math. Soc. (JEMS) 14 (2012), 1565-1597.

[20] Haberl, C., and Parapatits, L. The centro-affine Hadwiger theorem. J. Amer. Math. Soc. 27 (2014), 685-705.

[21] Haberl, C., And Parapatits, L. Moments and valuations. Amer. J. Math. 138 (2017), 1575-1603.

[22] Hadwiger, H. Vorlesungen über Inhalt, Oberfläche und Isoperimetrie. Springer, Berlin, 1957.

[23] Huang, Y., Lutwak, E., Yang, D., And Zhang, G. Geometric measures in the dual Brunn-Minkowski theory and their associated Minkowski problems. Acta Math. 216 (2016), 325-388.

[24] Klain, D. A., And Rota, G.-C. Introduction to Geometric Probability. Cambridge University Press, Cambridge, 1997.

[25] Klartag, B., and Milman, V. Geometry of log-concave functions and measures. Geom. Dedicata 112 (2005), 169-182. 
[26] Kone, H. Valuations on Orlicz spaces and $L^{\phi}$-star sets. Adv. in Appl. Math. 52 (2014), 82-98.

[27] Li, J., And Leng, G. Orlicz valuations. Indiana Univ. Math. J. 66 (2017), 791-819.

[28] Li, J., And MA, D. Laplace transforms and valuations. J. Funct. Anal. 272 (2017), 738-758.

[29] Ludwig, M. Valuations on polytopes containing the origin in their interiors. Adv. Math. 170 (2002), 239-256.

[30] Ludwig, M. Fisher information and matrix-valued valuations. Adv. Math. 226 (2011), 2700-2711.

[31] Ludwig, M. Valuations on function spaces. Adv. Geom. 11 (2011), 745-756.

[32] Ludwig, M. Valuations on Sobolev spaces. Amer. J. Math. 134 (2012), 824-842.

[33] Ludwig, M. Covariance matrices and valuations. Adv. in Appl. Math. 51 (2013), 359-366.

[34] Ludwig, M., And Reitzner, M. A classification of $\operatorname{SL}(n)$ invariant valuations. Ann. of Math. (2) 172 (2010), 1219-1267.

[35] Ludwig, M., And Reitzner, M. SL(n) invariant valuations on polytopes. Discrete Comput. Geom. 57 (2017), 571-581.

[36] Ma, D. Real-valued valuations on Sobolev spaces. Sci. China Math. 59 (2016), 1-14.

[37] McMullen, P. Metrical and Combinatorial Properties of Convex Polytopes. In Proc. Int. Congr. Math. (1975), R. James, Ed., Canadian Mathematical Congress, pp. 491-495.

[38] McMullen, P. Valuations and Euler type relations on certain classes of convex polytopes. Proc. London Math. Soc. 35 (1977), 113-135.

[39] Milman, V., And Rotem, L. Mixed integrals and related inequalities. J. Funct. Anal. 264 (2013), $570-604$.

[40] Ober, M. $L_{p}$-Minkowski valuations on $L^{q}$-spaces. J. Math. Anal. Appl. 414 (2014), 68-87.

[41] Rockafellar, R. T. Convex Analysis. Princeton University Press, 1970.

[42] Rockafellar, R. T., And Wets, R. J.-B. Variational Analysis, 3rd ed., vol. 317 of Grundlehren der mathematischen Wissenschaften. Springer-Verlag, Berlin, 2009.

[43] Schneider, R. Convex Bodies: The Brunn-Minkowski Theory, Second expanded ed., vol. 151 of Encyclopedia of Mathematics and its Applications. Cambridge University Press, Cambridge, 2014.

[44] Schuster, F. E., And Wannerer, T. Minkowski valuations and generalized valuations. J. Eur. Math. Soc. $(J E M S)$ (in press).

[45] Tradacete, P., And Villanueva, I. Continuity and representation of valuations on star bodies. Adv. Math. 329 (2018), 361-391.

[46] Tsang, A. Valuations on $L_{p}$-spaces. Int. Math. Res. Not. IMRN 20 (2010), 3993-4023.

[47] Tsang, A. Minkowski valuations on $L_{p}$-spaces. Trans. Amer. Math. Soc. 364 (2012), 6159-6186.

[48] Villanueva, I. Radial continuous rotation invariant valuations on star bodies. Adv. Math. 291 (2016), 961-981.

[49] Wang, T. Semi-valuations on BV $\left(\mathbb{R}^{n}\right)$. Indiana Univ. Math. J. 63 (2014), 1447-1465.

Fabian Mussnig

Institut für Diskrete Mathematik und Geometrie

Technische Universität Wien

Wiedner Hauptstraße 8-10/1046

1040 Wien, Austria

e-mail: fabian.mussnig@tuwien.ac.at 